US Army Corps

of Engineers ${ }_{\circledast}$

Engineer Research and

Development Center

Flood and Coastal Systems Research Program

Theory, Formulation, and Implementation of the Cartesian and Spherical Coordinate TwoDimensional Depth-Averaged Module of the Adaptive Hydraulics (AdH) Finite Element Numerical Code

Gaurav Savant, Charlie Berger, Corey J. Trahan, and Gary L. Brown 
The US Army Engineer Research and Development Center (ERDC) solves the nation's toughest engineering and environmental challenges. ERDC develops innovative solutions in civil and military engineering, geospatial sciences, water resources, and environmental sciences for the Army, the Department of Defense, civilian agencies, and our nation's public good. Find out more at www.erdc.usace.army.mil.

To search for other technical reports published by ERDC, visit the ERDC online library at http://acwc.sdp.sirsi.net/client/default. 


\title{
Theory, Formulation, and Implementation of the Cartesian and Spherical Coordinate Two- Dimensional Depth-Averaged Module of the Adaptive Hydraulics (AdH) Finite Element Numerical Code
}

\author{
Gaurav Savant, Charlie Berger, and Gary L. Brown \\ Coastal and Hydraulics Laboratory \\ US Army Engineer Research and Development Center \\ 3909 Halls Ferry Road \\ Vicksburg, MS 39180-6199 \\ Corey J. Trahan \\ Information Technology Laboratory \\ US Army Engineer Research and Development Center \\ 3909 Halls Ferry Road \\ Vicksburg, MS 39180-6199
}

Final report

Approved for public release; distribution is unlimited.

Prepared for Flood and Coastal Systems Research and Development Program US Army Engineer Research and Development Center 3909 Halls Ferry Road

Vicksburg, MS 39180-6199

Under Funding Acct Code U4354615; Work Unit Adaptive Hydraulics (AdH)

Development, Verification, and Validation 


\section{Abstract}

The US Army Engineer Research and Development Center, Coastal and Hydraulics Laboratory, has undertaken the development of the multimodule Adaptive Hydraulics (AdH) hydrodynamic, sediment, water quality, and transport numerical code. This report documents the mathematical formulation and numerical implementation of the twodimensional depth-averaged module of AdH.

DISCLAIMER: The contents of this report are not to be used for advertising, publication, or promotional purposes. Citation of trade names does not constitute an official endorsement or approval of the use of such commercial products. All product names and trademarks cited are the property of their respective owners. The findings of this report are not to be construed as an official Department of the Army position unless so designated by other authorized documents. 


\section{Contents}

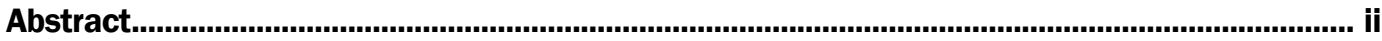

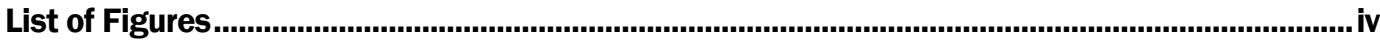

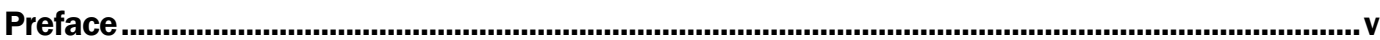

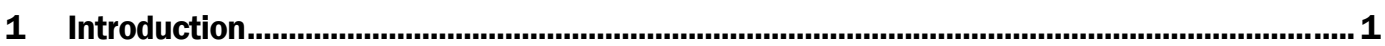

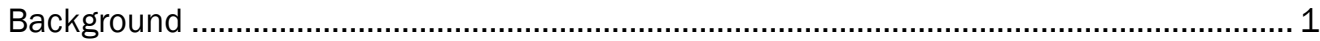

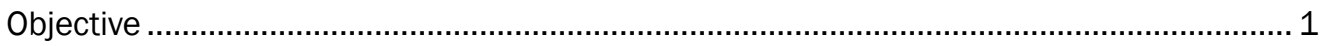

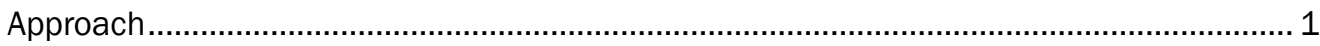

2 Depth-Averaged Shallow Water Equations in the Cartesian Coordinate System................... 3

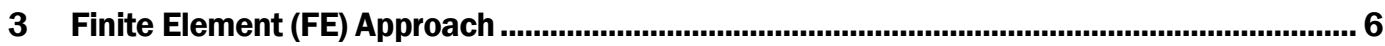

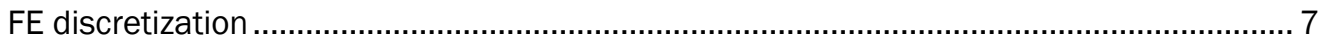

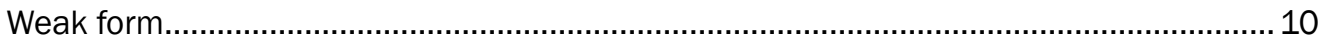

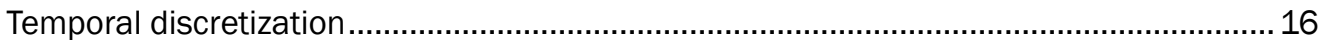

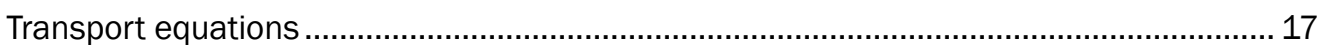

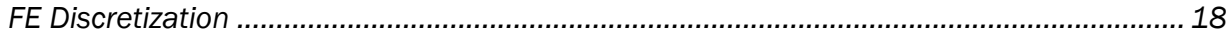

Temporal discretization ........................................................................................ 18

4 Depth-Averaged Shallow Water Equations in the Spherical Coordinate System ..................19

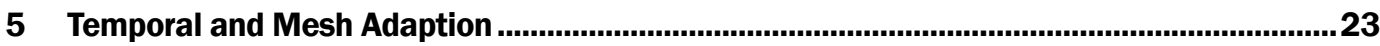

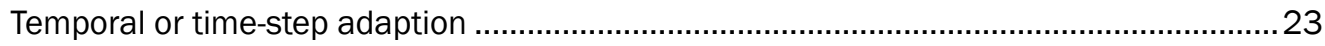

Failure-Indicated time-step adaption ............................................................................ 23

Pseudo-Transient Computation (PTC) and Switched Evolution Relaxation (SER) .................23

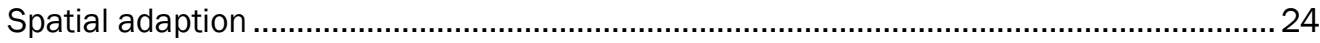

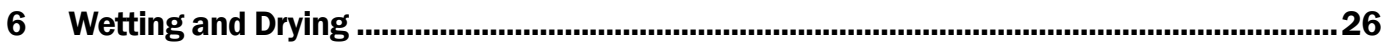

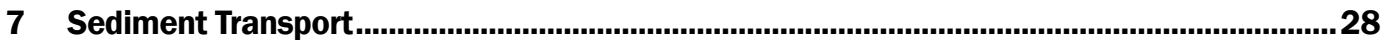

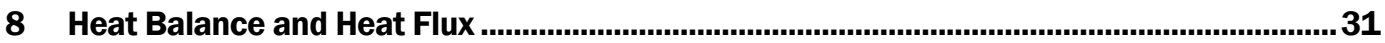

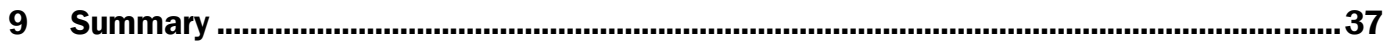

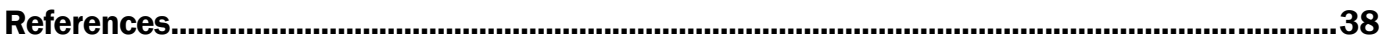

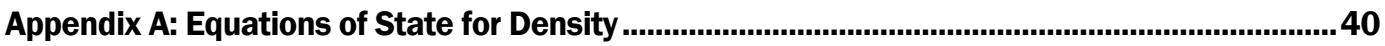

Appendix B: Discretization and Integration of Individual Terms ...............................................

Appendix C: Friction Library..............................................................................................................61

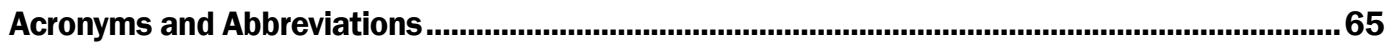

Report Documentation Page 


\section{List of Figures}

\section{Figures}

Figure 1. Computational element figure. .................................................................................. 8

Figure 2. Variation in $\zeta$ and $\eta$.............................................................................................. 8

Figure 3. Constants for principal tidal constituents (Parker 2007) ............................................... 21

Figure 4. Refinement and un-refinement of an element. ............................................................... 25

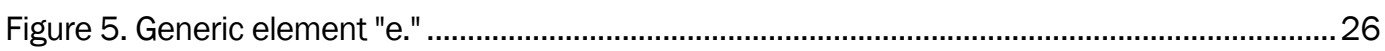

Figure 6. One node dry case. ...............................................................................................2 26

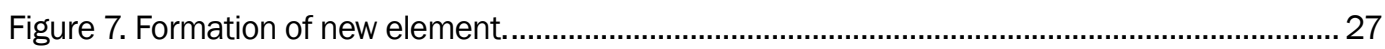

Figure 8. Sources and sinks of heat (adapted from Deas and Lowney [2000]).............................. 31

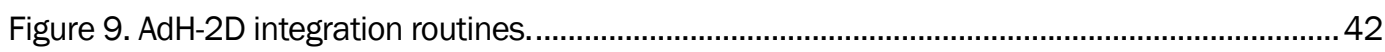

Figure 10. 1D Line integral basis functions............................................................................ 52

Figure 11. 1D line integral basis functions on 2D elements.........................................................58 


\section{Preface}

This study was conducted for the Flood and Coastal Systems (F\&CS) Research and Development (R\&D) Program under Work Unit Adaptive Hydraulics (AdH) Development, Verification and Validation; Funding Acct Code U4354615. F\&CS R\&D is funded by the Flood and Coastal Risk Management (F\&CRM) business line of the Headquarters, US Army Corps of Engineers (HQUSACE), and is administered by the US Army Engineer Research and Development Center (ERDC), Coastal and Hydraulics Laboratory (CHL), Vicksburg, MS, under the Flood Risk Management Program of HQUSACE. Mr. Mark S. Roupas, HQUSACE, and Mr. Jeffrey Jensen, USACE Institute for Water Resources, jointly manage the F\&CRM Business Line. Dr. Julie Dean Rosati was the Technical Director for F\&CRM Research and Development at the time of this study.

Technical reviews and discussions of this report were provided by Dr. Matthew Farthing and Dr. Yan Ding of ERDC-CHL. At the time of publication of this report, oversight and guidance of this work was provided by Mr. Keith Flowers, Chief, Estuarine and Riverine Engineering Branch, and Dr. Cary Talbot, Chief, Flood and Coastal Engineering Division. Mr. Jeffrey R. Eckstein was the Deputy Director of CHL, and Dr. Ty V. Wamsley was the Director.

COL Teresa A. Schlosser was ERDC Commander, and Dr. David Pittman was ERDC Director. 


\section{Introduction}

The US Army Corps of Engineers (USACE), through the US Army Engineer Research and Development Center, has developed a robust, multi-dimensional, and mass conservative finite element (FE) hydrodynamic and constituent transport numerical model, Adaptive Hydraulics. Adaptive Hydraulics has been referred to as "ADH" and "AdH" in literature; the abbreviation $\mathrm{AdH}$ is used in this report in accordance with how Adaptive Hydraulics is referenced in peer-reviewed literature.

\section{Background}

The USACE hydrodynamic and transport numerical code AdH has been developed to be a general purpose hydrodynamic model over the past several years. As a normal consequence of this development effort, the underlying mathematics and numerical techniques require documentation. This documentation is required to not only provide a reference for users of the code but for the developers themselves to allow the maintenance of the most efficient and error-free code possible.

\section{Objective}

The objective of this report is to succinctly and accurately provide the mathematical and numerical background underpinning the $\mathrm{AdH}$ numerical code.

\section{Approach}

The objectives of this report are to provide the mathematical and numerical basis of the two-dimensional (2D) shallow water module of AdH (AdH-2D). This report obtains this objective by providing the formulation and discretization in distinct chapters as follows:

1. Chapter 2 provides the generic $2 \mathrm{D}$ shallow water equations, including salient points that distinguish the mass conservative 2D shallow water equations from the non-conservative $2 \mathrm{D}$ shallow water equations.

2. Chapter 3 provides detailed FE discretization, including a description of the stabilization scheme implemented into AdH-2D. 
3. Chapter 4 provides details of the modifications to the Cartesian coordinate shallow water equations to enable AdH-2D to perform Spherical co-ordinate simulations using Cartesian master elements.

4. Chapter 5 describes the dynamic run-time temporal and mesh adaption schemes implemented into AdH-2D.

5. Chapter 6 describes the mass conservative front tracking wet-dry scheme implemented into AdH-2D.

6. Chapter 7 describes the linkage of AdH-2D to the sediment transport library, SEDLIB.

7. Chapter 8 describes, in detail, the heat transfer formulations implemented into AdH-2D.

8. Chapter 9 provides a summary of this report.

9. Appendix A describes the equations of state for salinity, heat and sediment implemented into AdH-2D.

10. Appendix B describes in detail the FE discretization of each individual term in the shallow water equations, including the discretization of boundary integration terms, and the treatment of stabilization terms.

11. Appendix $\mathrm{C}$ provides a basic description of the friction library implemented into AdH-2D. 


\section{Depth-Averaged Shallow Water Equations in the Cartesian Coordinate System}

The AdH-2D in Cartesian Coordinates module of the AdH system has been in use since approximately 2003. It has been applied in hundreds of applications; a few representative examples are Berger and Lee (2004); McAlpin et al. (2009); and Bislip-Morales and Stockstill (2013). It is included here along with all of the newer features for completeness. The AdH-2D model utilizes the depth-averaged, Reynolds Averaged NavierStokes (RANS) equations under the assumption that (1) the horizontal length scale is much greater than the vertical length scale and (2) the pressure is hydrostatic. The model is routinely used to compute unsteady hydrodynamics in water bodies such as oceans, estuaries, rivers, reservoirs, marshes, etc. The assumption of a hydrostatic water column reduces the RANS equations to the well-known 2D shallow water (SW) equations. In these equations, the conservation of mass and momentum for a continuum of incompressible fluid is mathematically described by the continuity and momentum equations. In two dimensions, these equations are described as

$$
\bar{R}^{*} \equiv \frac{\partial \boldsymbol{Q}}{\partial t}+\frac{\partial \boldsymbol{F}_{x}}{\partial x}+\frac{\partial \boldsymbol{F}_{y}}{\partial y}+\boldsymbol{S}=0
$$

where

$$
\begin{gathered}
\boldsymbol{Q}=\left\{\begin{array}{l}
h \\
\bar{u} h \\
\bar{v} h
\end{array}\right\} \\
\boldsymbol{F}_{x}=\left\{\begin{array}{l}
\bar{u} h \\
\bar{u} h+\frac{1}{2} \frac{\rho g h^{2}}{\rho_{0}}-h \frac{\sigma_{x x}}{\rho_{0}} \\
\overline{u v} h-h \frac{\sigma_{y x}}{\rho_{0}}
\end{array}\right\}
\end{gathered}
$$




$$
\boldsymbol{F}_{y}=\left\{\begin{array}{l}
\bar{v} h \\
\bar{u} \bar{v} h-h \frac{\sigma_{x y}}{\rho_{0}} \\
\bar{v} h+\frac{1}{2} \frac{\rho g h^{2}}{\rho_{0}}-h \frac{\sigma_{y y}}{\rho_{0}}
\end{array}\right\}
$$

and

$$
\boldsymbol{S}=\left\{\begin{array}{l}
-r \\
\frac{\rho}{\rho_{0}} g h \frac{\partial z_{b}}{\partial x}+\frac{h}{\rho_{0}} \frac{\partial P}{\partial x}+\frac{1}{2} C_{f} \bar{u}\|V\|^{1 / 2}-\frac{\tau_{x_{-} s}}{\rho_{0}}-f \bar{v} h \\
\frac{\rho}{\rho_{0}} g h \frac{\partial z_{b}}{\partial y}+\frac{h}{\rho_{0}} \frac{\partial P}{\partial y}+\frac{1}{2} C_{f} \bar{v}\|V\|^{1 / 2}-\frac{\tau_{y_{-} s}}{\rho_{0}}+f \bar{u} h
\end{array}\right\}
$$

The following definitions complete these equations:

$$
\begin{aligned}
H & =\text { depth of fluid } \\
\bar{u}, \bar{v} & =\text { depth-averaged velocity in the } x \text { and } y \text { directions, respectively } \\
r & =\text { rainfall/evaporation } \\
\rho & =\rho(x, y, t) \text { is the fluid density } \\
\rho_{0} & =\text { reference density } \\
g & =\text { acceleration due to gravity } \\
z_{b} & =\text { bottom elevation }
\end{aligned}
$$

$\sigma_{x x}, \sigma_{y y}, \sigma_{x y}$ and $\sigma_{y x}=$ Reynolds stress in $x x, y y, x y$ and $y x$ direction, respectively

$\tau_{x_{-} s}$ and $\tau_{y_{-} s}=$ wind or wave surface stresses in the $x$ and $y$ direction, respectively

$C_{f}=$ bottom friction coefficient

$F=$ Coriolis coefficient $=2 \omega \sin (\theta)$

$$
\begin{aligned}
\|V\|^{1 / 2} & =\sqrt{\bar{u}^{2}+\bar{v}^{2}}, \\
\omega & =\text { angular rotation of the Earth } \\
\theta & =\text { latitude } \\
P & =\text { pressure at the water surface. }
\end{aligned}
$$


To minimize notation, the bar over the depth-averaged values will be dropped in the remaining sections, and it is assumed that all velocities are depth averaged.

Reynolds stresses are implemented using the streamwise and transverse set of coefficients:

$$
\begin{gathered}
\sigma_{x x}=2 v_{s t} n_{v x}\left(n_{v x} \frac{\partial u}{\partial x}+n_{v y} \frac{\partial u}{\partial y}\right)+2 v_{t r} \frac{\partial u}{\partial x} \\
\sigma_{x y}=v_{s t} n_{v y}\left(n_{v y} \frac{\partial u}{\partial y}+n_{v x} \frac{\partial u}{\partial y}+n_{v x} \frac{\partial v}{\partial x}+n_{v y} \frac{\partial v}{\partial y}\right)+v_{t r}\left(\frac{\partial u}{\partial y}+\frac{\partial v}{\partial x}\right) \\
\sigma_{y x}=v_{s t} n_{v x}\left(n_{v y} \frac{\partial u}{\partial y}+n_{v x} \frac{\partial u}{\partial x}+n_{v x x} \frac{\partial v}{\partial x}+n_{v y} \frac{\partial v}{\partial y}\right)+v_{t r}\left(\frac{\partial u}{\partial y}+\frac{\partial v}{\partial x}\right) \\
\sigma_{y y}=2 v_{s t} n_{v y}\left(n_{v x} \frac{\partial v}{\partial x}+n_{v y} \frac{\partial v}{\partial y}\right)+2 v_{t r} \frac{\partial v}{\partial x}
\end{gathered}
$$

where

$$
\begin{aligned}
& n_{v x}=\frac{u}{\|V\|^{1 / 2}} \\
& n_{v y}=\frac{v}{\|V\|^{1 / 2}}
\end{aligned}
$$

$v_{s t}=$ kinematic turbulent eddy viscosity in the streamwise direction

$v_{t r}=$ kinematic turbulent eddy viscosity in the transverse direction.

Note on conservative and non-conservative forms of the shallow water equation

Equation 1 is written in what is generally referred to as the conservative form of the shallow water equations. If the momentum terms are expanded using the product rule and eliminating contributions that denote mass conservation, they transform to the non-conservative form. The non-conservative form of the equations do not conserve momentum across shocks or hydraulic jumps; this is an obvious consequence of the fact that velocities are not subject to any fundamental conservation principle in this form. 


\section{Finite Element (FE) Approach}

Numerical modeling of advection-diffusion equations has a long history in the field of applied mathematics. Methods that are most employed in riverine and ocean modeling are finite difference, finite volume, finite, and spectral-element techniques. The chief advantage of the FE method over the finite difference method is the ability to deal with unstructured grids. This kind of grid is attractive for numerical modelers for a number reasons. First, it facilitates the creation of irregular domains, such as uneven coastlines, narrow straits, and islands often found in oceanography as well as uneven river banks. Unstructured grids also offer the flexibility of spatially varying resolutions so that, for example, regions of interest can be extremely high resolution and vice versa. Therefore, computational effort is concentrated where the flow necessitates it. Finally, structured grids based on the geographical coordinates are plagued with difficulties near the poles. This is due to the convergence of the meridians, which requires unacceptably small time-steps to maintain stability. This constraint is not as much of an issue with unstructured grids.

In addition to these benefits, the $\mathrm{FE}$ method also rests on a rigorous mathematical framework based on a weighted residuals formulation that permits a precise definition of notions such as the error, convergence rate, and stability conditions. Last, natural boundary conditions are easily applied as they enter the weak statement of the problem directly with no further impositions or approximations.

A critical issue when applying the FE method to the shallow water equations is to find a suitable $\mathrm{FE}$ discretization pair for velocity and surface elevation (pressure). This pair should represent the geophysical flow accurately, be mass preserving, and not allow locking or the existence of spurious computational modes. AdH-2D utilizes triangular elements that have been shown to lock for elemental constant pressures with linear velocity. Locking is a result of the continuity equation being enforced too much as a result of the presence of more continuity equations (one per element) compared to the number of momentum equation pairs (one per node). The locking means that the velocity will be forced to a uniform value of zero. (For an explanation of the locking phenomena, see Carey and Oden [1986]). Linear pressure and linear velocity typically face problems with spurious modes. These spurious modes have a wide range of characteristics and may take the form of pressure (surface-elevation), 
velocity, and/or Coriolis modes. The modes may cause aliasing and an accumulation of energy in the smallest-resolvable scale, leading to noisy solutions (see Walters and Carey [1984]).

An early attempt to eliminate the spurious oscillations found in the Galerkin FE SW formulation was to use mixed interpolation schemes for pressure (elevation) and velocities, with the pressure discretization at lower order (typically linear pressure and quadratic velocity). Although this mitigated the pressure oscillations, the velocity issues still remained. Throughout the years, a number of alternative methods have been introduced to reduce these oscillations, such as making use of the Generalized Wave Continuity Equation (Luettich and Westerink 2004) or by using a discontinuous Galerkin method. However, since a priority mission of the AdH-2D model is to accurately simulate transcritical, supercritical, and subcritical flows, it was decided that the same Streamline Upwind Petrov-Galerkin (SUPG) scheme, successfully used in the previous shallow water model HIVEL2D, would be used as a basis for AdH-2D.

Unlike the standard Galerkin method, in the SUPG method (Hughes and Brook 1982) test/weight and trial functions are not the same. In the SUPG method, the test function is a linear combination of the trial function and some weighting of the spatial derivative of the trial function. This additional, elementally discontinuous, trial gradient term suppresses spatial oscillations in pressure and velocity in a controllable and consistent manner. Importantly, the SUPG method is not simply the Galerkin method with stabilization; it is a modification of the test function in a manner that is discretely consistent with the original equations of motion. The specific AdH-2D SUPG method used was originally described in Stockstill and Berger (1994).

\section{FE discretization}

The FE discretization is performed over a triangular element mapped to a computational parent $\{(0,0) ;(0,1) ;(1,0)\}$ as represented in Figures 1 and 2.

The 2D linear basis functions, denoted as $\phi_{i}$, used for trial function discretization, are defined nodally on the parent element as 


$$
\begin{aligned}
& \phi_{1}=1-\xi-\eta \\
& \phi_{2}=\xi \\
& \phi_{3}=\eta
\end{aligned}
$$

where

$\xi$ and $\eta$ are locally defined variables whose values vary linearly from o to 1 (Figure 2) so that

1. at node 1 , the value of $\xi$ and $\eta$ is 0 ; and therefore $\phi_{1}$ is equal to $1, \phi_{2}$ and $\phi_{3}$ are equal to 0

2. at node 2, the value of $\xi$ is 1 and $\eta$ is 0 ; and therefore $\phi_{2}$ is equal to $1, \phi_{1}$ and $\phi_{3}$ are equal to $\mathrm{o}$

3. at node 3 , the value of $\xi$ is 0 and $\eta$ is 1 ; and therefore $\phi_{3}$ is equal to $1, \phi_{1}$ and $\phi_{2}$ are equal to 0 .

Figure 1. Computational element figure.

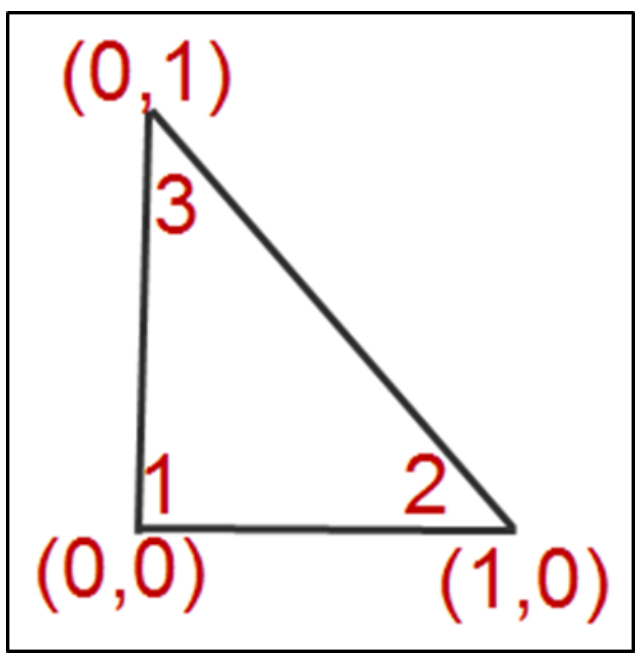

Figure 2. Variation in $\zeta$ and $\eta$.

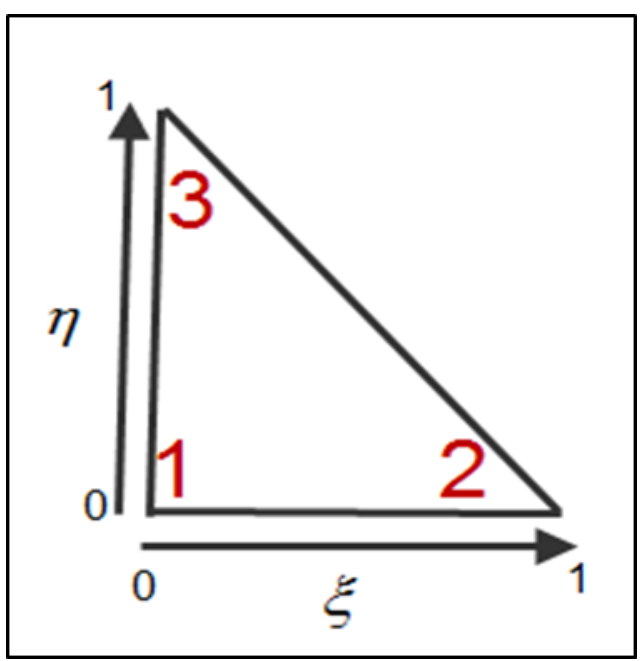

In traditional $\mathrm{FE}$ fashion, the linear trial functions are used to expand all solution variables so that for 3 node triangles, the continuous fields discretize to

$$
\begin{aligned}
& x=\sum_{1}^{3} x_{i} \phi_{i}(\xi, \eta)=x_{1} \phi_{1}(\xi, \eta)+x_{2} \phi_{2}(\xi, \eta)+x_{3} \phi_{3}(\xi, \eta) \\
& y=\sum_{1}^{3} y_{i} \phi_{i}(\xi, \eta)=y_{1} \phi_{1}(\xi, \eta)+y_{2} \phi_{2}(\xi, \eta)+y_{3} \phi_{3}(\xi, \eta)
\end{aligned}
$$




$$
\begin{aligned}
& u(\xi, \eta, t)=\sum_{1}^{3} u_{i}(\xi, \eta, t) \phi_{i}(\xi, \eta) \\
& v(\xi, \eta, t)=\sum_{1}^{3} v_{i}(\xi, \eta, t) \phi_{i}(\xi, \eta) \\
& h(\xi, \eta, t)=\sum_{1}^{3} h_{i}(\xi, \eta, t) \phi_{i}(\xi, \eta)
\end{aligned}
$$

where all variables are as previously defined.

Equations 14 through 16 above expand the variables in the computational or parent space. The expansion in physical space is written as

$$
\begin{aligned}
& u(x, y, t)=\sum_{1}^{3} u_{i}(x, y, t) \hat{\phi}_{i}(x, y) \\
& v(x, y, t)=\sum_{1}^{3} v_{i}(x, y, t) \hat{\phi}_{i}(x, y) \\
& h(x, y, t)=\sum_{1}^{3} h_{i}(x, y, t) \hat{\phi}_{i}(x, y)
\end{aligned}
$$

Where $u(x, y, t), v(x, y, t)$, and $h(x, y t)$ are time varying $x$-direction velocity, $y$-direction velocity, and depth, respectively. Hereafter, $u, v$, and $h$ will be used to refer to the time varying quantities. In these equations, $\hat{\phi}$ is the basis function in the physical space.

The transformation from the computational element to the physical element utilizes the Jacobian, $J$, defined as

$$
J(\xi, \eta)=\left(\begin{array}{ll}
\frac{\partial x}{\partial \xi} & \frac{\partial x}{\partial \eta} \\
\frac{\partial y}{\partial \xi} & \frac{\partial y}{\partial \eta}
\end{array}\right)
$$


The relationship between the physical element area, denoted as $\Lambda$, and $\mathrm{J}$ is

$$
\Lambda=\frac{1}{2}|J(\xi, \eta)|
$$

All FE integrations are performed on the computational element. The following equations, for example, are typical mass and stiffness transformations from the physical to parent domain physical element:

$$
M=\int_{\Omega_{e}^{2}} \hat{\phi}_{i}(x, y) \hat{\phi}_{j}(x, y) d \Omega=\int_{\xi=0}^{1} \int_{\eta=0}^{1-\xi} \phi_{i}(\xi, \eta) \phi_{j}(\xi, \eta)(2 \Lambda) d \xi d \eta
$$

and

$$
K=\int_{\Omega_{e}^{2 d}} \frac{\partial \hat{\phi}_{i}}{\partial x} \frac{\partial \hat{\phi}_{j}}{\partial y} d \Omega=\frac{1}{2 \Lambda} \int_{\xi=0}^{1} \int_{\eta=0}^{1-\xi}\left(\frac{\partial \phi_{i}}{\partial x}\right)\left(\frac{\partial \phi_{j}}{\partial y}\right) d \xi d \eta
$$

where

$$
\Omega_{e}^{2 d}=2 \mathrm{D} \text { elemental domain (hereafter, referred to as } \Omega_{e} \text { ) }
$$

\section{Weak form}

One begins with the weak form of the analytic equations and then progresses to the FE statement. The weak statement of equation set (1) is

$$
\int_{\Omega}\left(w \frac{\partial \mathbf{Q}}{\partial t}-\frac{\partial w}{\partial x} \mathbf{F}_{x}-\frac{\partial w}{\partial y} \mathbf{F}_{y}+w \mathbf{S}\right) d \Omega+\int_{\Gamma} w\left(\mathbf{F}_{x} n_{x}+\mathbf{F}_{y} n_{y}\right) d \Gamma=0
$$

The terms $n_{x}$ and $n_{y}$ are the outward normal direction components along the boundary $\Gamma$. The weight function (or test function), $w$, is a function that is sufficiently smooth so that the operations in the equation are integrable. Furthermore, $w$ must also enforce the Essential boundary conditions. The function $w$ is arbitrary except that it is zero along Essential boundary locations. Since $w$ is arbitrary, any solution to equation set (1) is also a solution to the weak form. If classical solutions exist, they will satisfy the weak form. If, however, only non-smooth 
solutions exist, the weak form will recover these and (1) will not. This is important for reproduction of hydraulic jumps and oblique shocks in the shallow water equations. Note that to this point exact solutions are being found. Now, there is a move to the FE statement in which numerical solutions that are approximations will be found.

The development of the FE statement will be made in a semi-discrete form with the temporal derivative remaining analytic. Thus, the development proceeds as a spatial interpolation. AdH SW2 is implicit, so the unknown variables are all written for the new time-step.

The dependent variables are interpolated on the individual elements using linear Lagrange polynomials.

$$
\begin{aligned}
& u_{h}(x, y, t)=\sum_{j} \phi_{j}(x, y) u_{j}(t) \\
& v_{h}(x, y, t)=\sum_{j} \phi_{j}(x, y) v_{j}(t) \\
& h_{h}(x, y, t)=\sum_{j} \phi_{j}(x, y) h_{j}(t)
\end{aligned}
$$

where the subscript " $j$ " indicates the nodal values of each variable.

Now, re-form Equation set (1) with these nodal variables such that all the terms should have a subscript " $h$. ." In the interest of simplicity, there will be a suspension from using the subscript " $h$ " to indicate the approximate or discrete values, but it should be understood that one is now dealing with the approximate equations.

Equation 1 provides the strong (or classical) form of the continuity and depth-averaged momentum equations; however, the FE method requires that these equations be formulated in their corresponding weak form by multiplying by a Petrov-Galerkin (PG) test/weight function, $\Psi=\phi+\varphi$, (trial + gradient function) and integrating by parts over the domain to give the following FE statement. 
For all $i$, find $u, v$, and $h$ such that

$$
\overline{\mathbf{R}_{i}} \equiv \sum_{e}\left[\left(\int_{\Omega_{e}} \phi_{i} \frac{\partial \mathbf{Q}}{\partial t}-\frac{\partial \phi_{i}}{\partial x} \mathbf{F}_{x}-\frac{\partial \phi_{i}}{\partial y} \mathbf{F}_{y}+\phi_{i} \mathbf{S}\right) d \Omega_{e}+\int_{\Gamma_{e} \cap \Gamma} \phi_{i}\left(\mathbf{F}_{x} n_{x}+\mathbf{F}_{y} n_{y}\right) d \Gamma_{e}+\overline{\mathbf{P}}_{i}\right]=\mathbf{0} \text { (26) }
$$

where

$\Omega_{\mathrm{e}}=$ elemental $2 \mathrm{D}$ domain

$\Gamma_{\mathrm{e}}=$ elemental boundary

$\Gamma=$ the global boundary

$n_{x}, n_{y}=$ unit outward normal in the $x$ - and $y$-directions, respectively.

$$
\overline{\mathbf{R}}_{i} \equiv\left\{\begin{array}{l}
\bar{R}_{c}^{i} \\
\bar{R}_{m x}^{i} \\
\bar{R}_{m y}^{i}
\end{array}\right\}
$$

$\overline{R_{c}^{i}}=$ residual of the $i^{\text {th }}$ discrete continuity equation

$\bar{R}_{m x}^{i}=$ residual of the $i^{\text {th }} x$-direction momentum equation

$\bar{R}_{m y}^{i}=$ residual of the $i^{\text {th }} y$-direction momentum equation

$$
\overline{\mathbf{P}}_{i} \equiv\left\{\begin{array}{l}
\bar{P}_{c}^{i} \\
\bar{P}_{m x}^{i} \\
\bar{P}_{m y}^{i}
\end{array}\right\}=\int_{\Omega_{e}} \tau^{e}\left[\mathbf{A}_{x}^{e} \frac{\partial \phi_{i}}{\partial x}+\mathbf{A}_{y}^{e} \frac{\partial \phi_{i}}{\partial y}\right] \overline{\mathbf{R}}_{n c}^{*} d \Omega_{e}
$$

$\bar{P}_{c}^{i}=$ SUPG contribution to the $i^{\text {th }}$ continuity equation

$\bar{P}_{m x}^{i}=$ SUPG contribution to the $i^{\text {th }} x$-direction momentum equation

$\bar{P}_{m y}^{i}=$ SUPG contribution to the $i^{\text {th }} y$-direction momentum equation $\boldsymbol{\tau}^{e}=$ stabilization parameter.

The discrete non-conservative shallow water equations are

$$
\overline{\boldsymbol{R}}_{n c}^{*}=\frac{\partial \mathbf{Q}_{n c}}{\partial t}+\mathbf{A}_{x} \frac{\partial \mathbf{Q}_{n c}}{\partial x}+\mathbf{A}_{y} \frac{\partial \mathbf{Q}_{n c}}{\partial y}+\mathbf{S}_{n c}=0
$$


where

$$
\begin{gathered}
\mathbf{Q}_{n c}=\left(\begin{array}{l}
h \\
u \\
v
\end{array}\right) \\
\mathbf{A}_{x}=\left[\begin{array}{lll}
u & h & 0 \\
\frac{\rho}{\rho_{o}} g & u & 0 \\
0 & 0 & u
\end{array}\right] \\
\mathbf{A}_{y}=\left[\begin{array}{lll}
v & 0 & h \\
0 & v & 0 \\
\frac{\rho}{\rho_{o}} g & 0 & v
\end{array}\right] \\
\mathbf{S}_{n c}=\left[\begin{array}{lll}
1 & 0 & 0 \\
0 & \frac{1}{h} & 0 \\
0 & 0 & \frac{1}{h}
\end{array}\right] \mathbf{S}
\end{gathered}
$$

The AdH-2D test function is then

$$
\Psi_{i} \equiv \mathbf{I} \phi_{i}+\tau^{e}\left[\mathbf{A}_{x}^{e} \frac{\partial \phi_{i}}{\partial x}+\mathbf{A}_{y}^{e} \frac{\partial \phi_{i}}{\partial y}\right]
$$

where

$$
\mathbf{I} \text { = identity matrix. }
$$

The superscript " $e$ " means an element average value. It is important that the gradient (or discontinuous) portion of the test function have elemental constant coefficients to be assured of elemental conservation. Another subtle point is that the discontinuous (SUPG) portion of the test function (termed $\varphi$ ) is only integrated against the discrete equation over the interior of the element. This means that Essential boundary conditions are 
addressed by the continuous portion of the test function. Also, the additional SUPG portion of the integration does not participate in enforcing flux jumps or natural boundary conditions. These are addressed by the continuous $\phi$ portion of the test function.

There are two driving principles in the development of the PGtest function. First, AdH should be as consistent as possible. That is, a test function is applied to the entire discrete equation set. The second guiding principle is simplicity. AdH is an implicit model that uses the Newton method to linearize the discrete equations. All additions that result in a decision within the code can adversely affect the convergence of the Newton method at times. Many methods that have a lot of decisions/branches were developed for static meshes. This is so the methods get the best result for a particular mesh. Instead, with AdH there is reliance upon adaptive refinement to produce stable and accurate solutions, and only utilize more complex numerical decisions when the results are substantially improved.

Consistency means that a test function is integrated with the entire discrete equations. This results in a higher-order accuracy (more accurately higherorder convergence rate). Examining equation set (24), one might suspect that the test function is not being applied to the same equation. First, the continuous test function portion is integrated by parts, and the discontinuous portion ( $\varphi$ portion) is not. Analytically, integration by parts and not integrating by parts are identical (for smooth conditions). The only difference arises if addressing non-smooth conditions when integration by parts allows the recovery of solutions with jumps. Note that the discontinuous portion of the test function is only applied within the element where the solution is smooth. Flux jumps are relegated to the element edges. The second issue is that the discontinuous test function is applied to the non-conservative equations set. This is done primarily for convenience and simplicity. For smooth conditions, as it is assumed within the element, non-conservative and conservative equations sets result in the same solutions. The one set of terms that is not included with the integration with the discontinuous portion of the test function is the diffusion terms. Since the diffusion terms are second-order spatial derivatives (and the depth is treated as a constant in these terms), the linear interpolation of velocity does not support two spatial derivatives. That is, the direct implementation of these terms in the discontinuous test function portion of the FE statement is zero. In effect, these diffusion terms are dropped from the 
discontinuous portion of the test function. This is a place in which the SUPG implementation consciously is inconsistent. These terms would be retained in the stabilization, however, if higher-order interpolation is used. And as one might expect, it is not a particularly good approach for simulating very low Reynolds number flow conditions.

Now consider the specific nature of the $\tau^{e}$ used in AdH-2D. This begins with the most general case and then is simplified to the form implemented in AdH-2D. Again, if a more complex form did not produce markedly better behavior or results, then it was not implemented in AdH-2D. In AdH-2D, as with most SUPG methods, this contribution is proportional to the gradient of the trial function, so that

$$
\varphi=\boldsymbol{\tau}^{e} \cdot \nabla \phi
$$

where

$$
\boldsymbol{\tau}^{\mathcal{e}}=\left[\begin{array}{c}
\tau_{x} \mathbf{A}_{x}^{e} \\
\tau_{y} \mathbf{A}_{y}^{e}
\end{array}\right]
$$

The choice of the stabilization parameters, $\boldsymbol{\tau}^{e}$, is now discussed. The most general form of the components are

$$
\tau_{i}=\frac{\alpha \gamma l_{e i}}{\left\|a_{i}\right\|}
$$

where the subscript " $i$ " indicates the two directions ( $\mathrm{X}$ and $\mathrm{Y}$ ), $\gamma$ is a blending function to account for low Reynolds number flow by turning off the PG contribution, $\alpha$ is a numerical coefficient (AdH-2D uses a standard range of $0<\alpha \leq 0.5$, with a default of 0.5 ), $l_{e i}$ is the element length in the $i^{\text {th }}$ direction, and $a_{i}$ is the spectral radius. The elemental length used in $\mathrm{AdH}-2 \mathrm{D}$ is given as

$$
l_{e i}=l_{e x}=l_{e y}=\sqrt{A_{e}}
$$

where $A_{e}$ is the area of the element under consideration. Again, this simplifying choice was made since the two-value length did not make markedly better results and AdH-2D can rely on adaption to improve 
solutions more simply. The blending function in AdH-2D is set to a constant of 1. AdH-2D applications are not low Reynolds number. However, it is very easy to implement this function if it becomes necessary.

AdH-2d defines the spectral radius as

$$
a^{e}=a_{x}=a_{y}=\sqrt{u^{2}+v^{2}+\frac{\rho}{\rho_{o}} g h}
$$

Therefore, the stabilization parameter is no longer a vector but instead a constant $\left(\boldsymbol{\tau}^{e}=\tau^{e}\right)$. The stabilization parameter can then be written as

$$
\tau^{e}=\alpha \frac{\sqrt{A_{e}}}{a^{e}}
$$

\section{Temporal discretization}

The temporal terms in the continuity and momentum equations are expressed using a second-order finite difference expression as

$$
\left(\frac{\partial h}{\partial t}\right)_{i}^{n+1}=\alpha_{t}\left[\frac{\left(\frac{3}{2} h_{i}^{n+1}-\frac{1}{2} h_{i}^{n}\right)-\left(\frac{3}{2} h_{i}^{n}-\frac{1}{2} h_{i}^{n-1}\right)}{\Delta t}\right]+(1-\alpha)\left[\frac{\left(h_{i}^{n+1}-h_{i}^{n}\right)}{\Delta t}\right]
$$

where

$$
\begin{aligned}
& \alpha_{t}=\text { factor that determines order of time stepping, } 0 \leq \alpha \leq 1 \\
& \Delta t=\text { time-step size } \\
& \mathrm{n} \text { indicates the } \mathrm{n}^{\text {th }} \text { time-step, so that the actual time is } t=n \Delta t .
\end{aligned}
$$

The $x$ - and $y$-direction momentum temporal terms are again expressed using a finite difference expression as

$$
\left(\frac{\partial h u}{\partial t}\right)_{i}^{n+1}=\alpha_{t}\left[\frac{\left(\frac{3}{2}(h u)_{i}^{n+1}-\frac{1}{2}(h u)_{i}^{n}\right)-\left(\frac{3}{2}(h u)_{i}^{n}-\frac{1}{2}(h u)_{i}^{n-1}\right)}{\Delta t}\right]+(1-\alpha)\left[\frac{\left((h u)_{i}^{n+1}-(h u)_{i}^{n}\right)}{\Delta t}\right]
$$


and

$$
\left(\frac{\partial h v}{\partial t}\right)_{i}^{n+1}=\alpha_{t}\left[\frac{\left(\frac{3}{2}(h v)_{i}^{n+1}-\frac{1}{2}(h v)_{i}^{n}\right)-\left(\frac{3}{2}(h v)_{i}^{n}-\frac{1}{2}(h v)_{i}^{n-1}\right)}{\Delta t}\right]+(1-\alpha)\left[\frac{\left((h v)_{i}^{n+1}-(h v)_{i}^{n}\right)}{\Delta t}\right]
$$

where all variables are as previously defined.

The exact forms of the integrated temporal terms in Equations 41-43 are presented in the appendices.

\section{Transport equations}

Adh-2D has the capability to transport passive as well as active constituents such as dyes, water quality constituents, salinity, temperature, etc., by weakly solving the advection-diffusion equation. This equation is written in the form of the mass conservation equation

$$
\frac{\partial h c}{\partial t}+\frac{\partial h u c}{\partial x}+\frac{\partial h v c}{\partial y}-\frac{\partial}{\partial x} D \frac{\partial c h}{\partial x}-\frac{\partial}{\partial y} D \frac{\partial c h}{\partial y}=0
$$

where

$$
\begin{aligned}
c & =\text { depth-averaged constituent concentration } \\
D & =\text { turbulent and molecular diffusivity. }
\end{aligned}
$$

Note that $u$ and $v$ are depth-averaged variables as well.

As was previously done with the shallow water equations, the transport equation is multiplied with the weight function and integrated to give the following weak form of Equation 44. 


\section{FE Discretization}

$$
\bar{R}_{t}=\sum_{e}\left[\begin{array}{l}
\int_{\Omega}\left(\begin{array}{l}
\frac{\phi_{i} \partial c h}{\partial t}-\frac{c h u \partial \phi_{i}}{\partial x}-\frac{c h v \partial \phi_{i}}{\partial y}+D_{x x} \frac{\partial h c}{\partial x} \frac{\partial \phi_{i}}{\partial x}+D_{x y} \frac{\partial h c}{\partial y} \frac{\partial \phi_{i}}{\partial x}+ \\
D_{y x} \frac{\partial h c}{\partial x} \frac{\partial \phi_{i}}{\partial y}+D_{y y} \frac{\partial h c}{\partial y} \frac{\partial \phi_{i}}{\partial y}
\end{array}\right) d \Omega \\
\phi_{\Gamma}\left(\begin{array}{l}
\left.(c h u) g \eta_{x}+(c h v) g_{y}-\left(D_{x x} \frac{\partial h c}{\partial x}\right) g \eta_{x}-\left(D_{x y} \frac{\partial h c}{\partial y}\right) g_{y}-\right) \\
\left(D_{y x} \frac{\partial h c}{\partial x}\right) g_{y}-\left(D_{y y} \frac{\partial h c}{\partial y}\right) g_{x} \\
+c_{e} \bar{P}_{c}+\bar{P}_{t}
\end{array}\right) d \Gamma+
\end{array}\right]
$$

Where $c_{e}$ is the elementally averaged concentration and $\bar{P}_{t}$ is the SUPG contribution to the transport equation defined in a way similar to the hydrodynamic continuity SUPG contribution. Note that to remain consistent with the depth-averaged continuity, the transport equation must also include its Petrov contribution term, $\bar{P}_{c}$.

\section{Temporal discretization}

The temporal term is again expressed using a backward Euler expression as

$$
\left(\frac{\partial h c}{\partial t}\right)_{i}^{n+1}=\alpha_{t}\left[\frac{\left(\frac{3}{2}(h c)_{i}^{n+1}-\frac{1}{2}(h c)_{i}^{n}\right)-\left(\frac{3}{2}(h c)_{i}^{n}-\frac{1}{2}(h c)_{i}^{n-1}\right)}{\Delta t}\right]+(1-\alpha)\left[\frac{\left((h c)_{i}^{n+1}-(h c)_{i}^{n}\right)}{\Delta t}\right]
$$

where all variables are as previously defined. 


\section{Depth-Averaged Shallow Water Equations in the Spherical Coordinate System}

AdH-2D has the capability to solve the equations for mass conservation and motion in the spherical coordinate by transforming them into Cartesian coordinates using the Carte Parallelo-grammatique projection.

Assuming that radius of the earth is much greater than the depth of the ocean and defining the following,

$$
\begin{aligned}
& x=\left(\lambda-\lambda_{0}\right) R \cos \phi_{0} \\
& y=R \phi
\end{aligned}
$$

where

$$
\begin{aligned}
\lambda & =\text { longitude } \\
\phi & =\text { latitude } \\
\lambda_{0} & =\text { longitude of reference point } \\
\phi_{0} & =\text { latitude of reference point } \\
R & =\text { radius of the Earth. }
\end{aligned}
$$

The following relationship can be written as

$$
\begin{aligned}
& \frac{\partial}{\partial \lambda}=\frac{\partial}{\partial x} \frac{\partial x}{\partial \lambda}+\frac{\partial}{\partial y} \frac{\partial y}{\partial \lambda}=R \cos \phi_{0} \frac{\partial}{\partial x} \\
& \frac{\partial}{\partial \phi}=\frac{\partial}{\partial x} \frac{\partial x}{\partial \phi}+\frac{\partial}{\partial y} \frac{\partial y}{\partial \phi}=R \frac{\partial}{\partial y}
\end{aligned}
$$

The depth-averaged continuity equation under the hydrostatic assumption in spherical coordinates can be written as

$$
\frac{\partial h}{\partial t}+\frac{1}{R \cos \phi} \frac{\partial h u}{\partial \lambda}+\frac{1}{R} \frac{\partial h v}{\partial \phi}-\frac{h v \tan \phi}{R}=0
$$

If a variable, $S$, is defined as follows,

$$
S=\frac{\cos \phi_{0}}{\cos \phi}
$$


Equation 49 can now be written as

$$
\frac{\partial h}{\partial t}+S \frac{\partial h u}{\partial x}+\frac{\partial h v}{\partial y}-\frac{h v \tan \phi}{R}=0
$$

The $x$-direction strong momentum equation is written as

$$
\begin{aligned}
& \frac{\partial h u}{\partial t}+\frac{1}{R \cos \phi} \frac{\partial h u u}{\partial \lambda}+\frac{1}{R} \frac{\partial h u v}{\partial \phi}-\frac{u v h \tan \phi}{R}-2 \omega v h \sin \phi+ \\
& \frac{1}{2 R \cos \phi} g \frac{\partial h^{2}}{\partial \lambda}-\frac{h}{R \cos \phi} \frac{\partial}{\partial \lambda}\left(\frac{1}{R \cos \phi} 2 \rho v \frac{\partial u}{\partial \lambda}\right)- \\
& \frac{h}{R} \frac{\partial}{\partial \phi} 2 \rho v\left[\frac{1}{R} \frac{\partial u}{\partial \phi}+\frac{1}{R \cos \phi} \frac{\partial v}{\partial \lambda}\right]+\frac{g h}{R \cos \phi} \frac{\partial z}{\partial \lambda}+\frac{\gamma}{R \cos \phi} \frac{\partial F}{\partial \lambda}=0
\end{aligned}
$$

where

$$
F=\alpha \sum_{n} C_{n} f_{n} L_{j} \cos \left[w_{n} t+j \lambda+K_{n}\right]
$$

with

$$
\begin{aligned}
F & =\text { tidal potential } \\
C n & =\text { Amplitude of tidal constituent " } n \text { " (Figure } 3) \\
f n & =\text { nodal factor for tidal constituent " } n \text { " } \\
K n & =\text { phase of tidal constituent " } n " \\
\alpha & =\text { elasticity of Earth }=0.69 \text { (Hendershott 1981) } \\
w_{n} & =\text { period of tidal component " } n \text { " (Figure } 3) \\
j & =0,1,2(j=\text { o declinational, }=1 \text { diurnal, }=2 \text { semidurnal) } \\
L_{O} & =3 \sin 2(\phi)-1 \\
L_{1} & =\sin (2 \phi) \\
L_{2} & =\cos ^{2}(\phi) .
\end{aligned}
$$


Figure 3. Constants for principal tidal constituents (Parker 2007).

\begin{tabular}{|cccc|}
\hline Species 'j' & Constituent & $\begin{array}{c}\text { C } \\
\text { (m) }\end{array}$ & $\begin{array}{c}\text { Phase } \\
\text { (o/hour) }\end{array}$ \\
\hline 0 & Mf & 0.041742 & 1.0980331 \\
& Mm & 0.022026 & 0.5443747 \\
& Ssa & 0.019446 & 0.0821373 \\
& Sa & $* *$ & 0.0410686 \\
\hline 1 & K1 & 0.141565 & 15.0410686 \\
& O1 & 0.100514 & 13.9430356 \\
& P1 & 0.046843 & 14.9589314 \\
& Q1 & 0.019256 & 13.3986609 \\
\hline 2 & M2 & 0.242334 & 28.9841042 \\
& S2 & 0.112841 & 30.000000 \\
& N2 & 0.046398 & 28.4397295 \\
& K2 & 0.030704 & 30.0821373 \\
\hline
\end{tabular}

(** heavily dependent on ocean state)

The $x$-direction strong momentum is further simplified to

$$
\begin{aligned}
& \frac{\partial h u}{\partial t}+S \frac{\partial h u u}{\partial x}+\frac{\partial h u v}{\partial y}-\frac{u v h \tan \phi}{R}-2 \omega v h \sin \phi+ \\
& \frac{S g}{2} \frac{\partial h^{2}}{\partial x}-S^{2} h \frac{\partial}{\partial x}\left(2 \rho v \frac{\partial u}{\partial x}\right)-h \frac{\partial}{\partial y}\left[2 \rho v\left(\frac{\partial u}{\partial y}+S \frac{\partial v}{\partial x}\right)\right]+ \\
& S g h \frac{\partial z}{\partial x}+\gamma S \frac{\partial F}{\partial x}=0
\end{aligned}
$$

The $y$-direction strong momentum equation can be similarly written as

$$
\begin{aligned}
& \frac{\partial h v}{\partial t}+\frac{1}{R \cos \phi} \frac{\partial h u v}{\partial \lambda}+\frac{1}{R} \frac{\partial h v v}{\partial \phi}+\frac{u u h \tan \phi}{R}+ \\
& 2 \omega u h \sin \phi+\frac{1}{2 R} g \frac{\partial h^{2}}{\partial \phi}-2 h \rho v \frac{1}{R} \frac{\partial v}{\partial \phi}- \\
& \frac{2 h \rho v}{R \cos \phi} \frac{\partial}{\partial \lambda}\left[\frac{1}{R} \frac{\partial u}{\partial \phi}+\frac{1}{R \cos \phi} \frac{\partial v}{\partial \lambda}\right]+\frac{g h}{R} \frac{\partial z}{\partial \phi}+\frac{\gamma}{R} \frac{\partial F}{\partial \phi}=0
\end{aligned}
$$

and

$$
\begin{aligned}
& \frac{\partial h v}{\partial t}+S \frac{\partial h v u}{\partial x}+\frac{\partial h v v}{\partial y}+\frac{u u h \tan \phi}{R}+2 \omega \sin \phi+\frac{1}{2} g \frac{\partial h^{2}}{\partial y} \\
& -\frac{h \partial}{\partial y}\left(2 \rho v \frac{\partial v}{\partial y}\right)-\frac{S h \partial}{\partial x}\left[2 \rho v\left(\frac{\partial u}{\partial y}+S \frac{\partial v}{\partial x}\right)\right]+g h \frac{\partial z}{\partial y}+\gamma \frac{\partial F}{\partial y}=0
\end{aligned}
$$


These equations are identical to the Cartesian equations presented in Equation 1, except for a modification to the $x$-gradient terms, which are now multiplied by the spherical correction factor, $S$. This factor can be linear expanded just as the solution variables to give

$$
S=\sum_{1}^{3} S_{i} \phi_{i}(\xi, \eta)=S_{1} \phi_{1}(\xi, \eta)+S_{2} \phi_{2}(\xi, \eta)+S_{3} \phi_{3}(\xi, \eta)
$$

The FE integrations in spherical coordinates are performed as explained in the previous sections; however, the SUPG spectral radius, $a_{i}$, is instead defined as

$$
a_{i}=a_{x}=a_{y}=\sqrt{S_{e}^{2}|U|^{2}+\frac{\rho}{\rho_{o}} g h}
$$

where $S_{e}$ is the elementally averaged spherical correction factor. 


\section{Temporal and Mesh Adaption}

One of the most widely used features of AdH-2D is the automatic temporal and spatial adaption options. In fact, it can be argued that temporal and spatial adaption is the only accurate way to adequately resolve transient flow and transport features. In this section, temporal and spatial adaption are discussed in detail.

\section{Temporal or time-step adaption}

Transient hydrodynamic and transport simulations usually require temporally varying time-steps, and a constant time-step size might not be sufficient to accurately capture the phenomenon of interest. To mitigate this, AdH-2D dynamically adapts the time-step using two formulations:

1. Failure-Indicated

2. Pseudo-Transient Steady State.

\section{Failure-Indicated time-step adaption}

AdH-2D tracks the number of non-linear iterations required to converge the solution to a user-specified tolerance. If the tolerance is not achieved within the maximum number of non-linear iterations specified, AdH-2D reduces the time-step and a new time-step is computed as

$$
d t^{\text {new }}=\frac{d t^{\text {old }}}{4}
$$

\section{Pseudo-Transient Computation (PTC) and Switched Evolution Relaxation (SER)}

Extremely transient hydrodynamic problems such as those that occur during dam and levee failure flows as well as in tidal bores can cause the "failure indicated time-step adaption" to go through several cycles of time-step reduction before arriving at an acceptable time-step size. For such problems, AdH-2D provides the user an option to use a modified PTC (Kelley and Keyes 1998) based time-step size computation. Savant et al. (2011) describe this modification as taking advantage of "initial accuracy of a, in general, temporally inaccurate computation after one newton iterate." The strategy used is called SER and is mathematically described as 


$$
d t^{n+1}=\min \left(d t^{n}\left\|\bar{R}_{c, m x, m y}^{n}\right\| /\left\|\bar{R}_{c, m x, m y}^{n+1}\right\|, d t_{\max }\right)
$$

where $\|$ indicates the 12 norm of the weak residual (given in Equation 26) after one newton iterate.

Equation 6o can result in a rapid increase in the time-step size, thereby jumping over important boundary events such as a singular wind stress event. To reduce the possibility of this occurring, AdH-2D modifies equation 60 to restrain the time-step size by always comparing against the initial time-step size. This is given as

$$
d t^{n+1}=\min \left(d t^{\text {initial }}\left\|\bar{R}_{c, m x, m y}^{\text {initial }}\right\| /\left\|\bar{R}_{c, m x, m y}^{n+1}\right\|, d t_{\max }\right)
$$

where all variables are as previously defined.

Savant et al. (2011) showed that the time-step size obtained through 61 is always the converged time-step. Importantly, the time-step size calculated through 61 does not guarantee that the simulation will achieve the specified tolerance within the maximum number of non-linear iterations specified. In these cases, AdH-2D reverts to the "failure indicated timestep adaption" for that time-step, and after a successful time-step, returns to the PTC-SER scheme.

\section{Spatial adaption}

Dynamic mesh adaption is an important and vital feature of AdH-2D, particularly for transient simulations such as tidal flows where a static mesh resolution may be inadequate or excessive. AdH-2D utilizes the mesh adaption strategy presented in Tate et al. (2006) to dynamically adapt the mesh. This strategy is based on an elemental error norm, given as

$$
E_{e}=\left(\int_{\Omega}\left[\frac{\partial h}{\partial t}+\frac{\partial h u}{\partial x}+\frac{\partial h v}{\partial y}\right]^{2} d \Omega\right) A_{e}
$$

where $A_{e}$ is the area of the $2 \mathrm{D}$ element. The indicator, $E_{e}$, is approximated numerically using residual values with integration points at each node of the element. If $E_{e}$ for an element is greater than the user specified limit, 
that element is flagged for refinement. The refinement process progresses as follows:

1. The longest edge is split.

2. Then the next longest edge is split.

3. The oldest edge is then split.

This process is repeated till the adaption reaches the maximum levels of refinement specified by the user. When the indicator falls below the userspecified error, the un-refinement process progresses in the order the adapted edges were formed. This process is illustrated below in Figure 4 for an element that is allowed to adapt twice.

Figure 4. Refinement and un-refinement of an element.

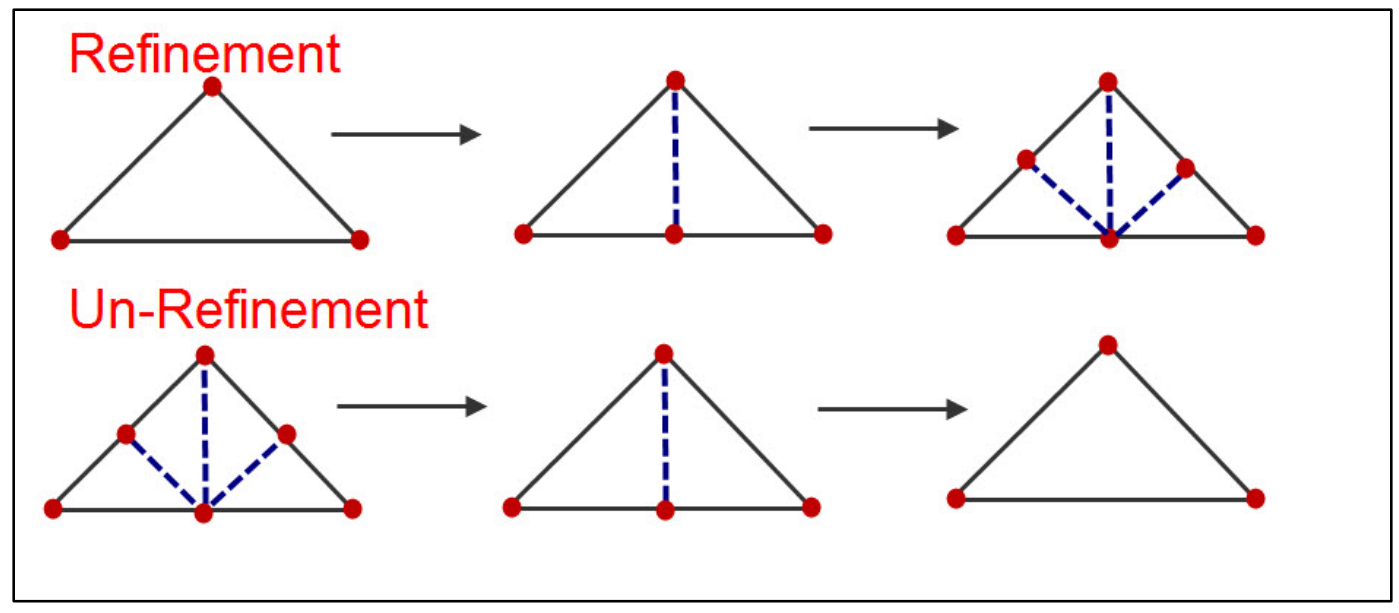




\section{Wetting and Drying}

Wetting and drying of a domain is an integral part of 2D modeling of rivers, dam breaks, and tidal systems. AdH-2D incorporates wetting and drying through a wet/dry front tracking algorithm.

Figure 5 illustrates a generic element "e."

Figure 5. Generic element "e."

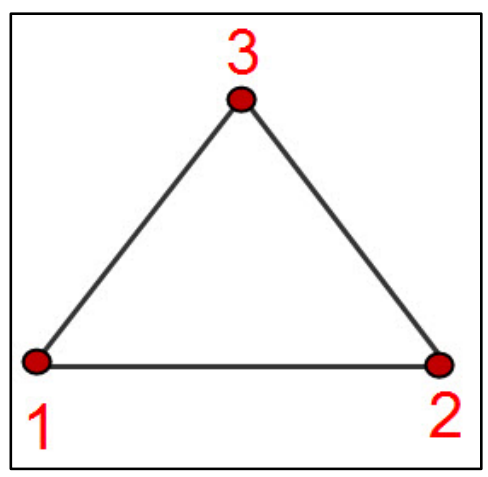

This element, while undergoing wetting or drying, has four possibilities:

1. Completely dry: in this, case no integration is performed on the element.

2. Completely wet: in this case, the entire element is integrated.

3. One node dry: in this case, the entire element is integrated as if it were completely wet; subsequently, the dry portion of the element is integrated (Figure 6), and this contribution is subtracted from the contribution of the entire element. In Figure 6, nodes 1 and 2 are wet, but node 3 is dry.

Figure 6. One node dry case.

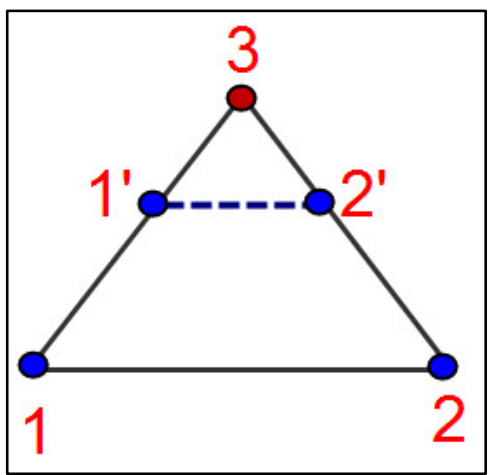


The dry element comprises of nodes 1', 2', and 3; the locations of nodes 1' and 2 ' are determined by locating the zero depth line by linearly interpolating from nodes 1 and 2.

4. Two nodes dry: in this case, a new element is formed (see Figure 7) by tracking the zero depth line and this new element is integrated.

Figure 7. Formation of new element.

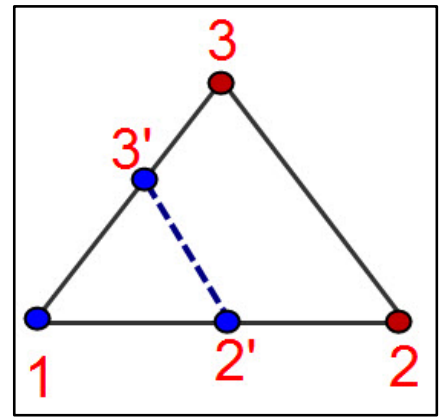

In Figure 7, the new element comprises of nodes 1, 2', and 3'. The position of nodes 2 ' and 3' are determined by linearly interpolating the location of the zero depth line. 


\section{Sediment Transport}

AdH-2D allows users to calculate the transport of cohesionless, cohesive and mixed sediments using implicit linkages to SEDLIB (Brown 2008). SEDLIB is designed to link to any code with an advection/diffusion solver. SEDLIB provides AdH-2D with sources and sinks for incorporation into its transport advection-diffusion equation solver. The solver then computes the suspended load transport (for silt and clay classes), bedload and suspended load transport (sand classes) and bedload transport (gravel classes). The sources and sinks are communicated to the parent code via a source/sink bed sediment flux for suspended load and bed load.

Equation 45 is modified for each grain class as

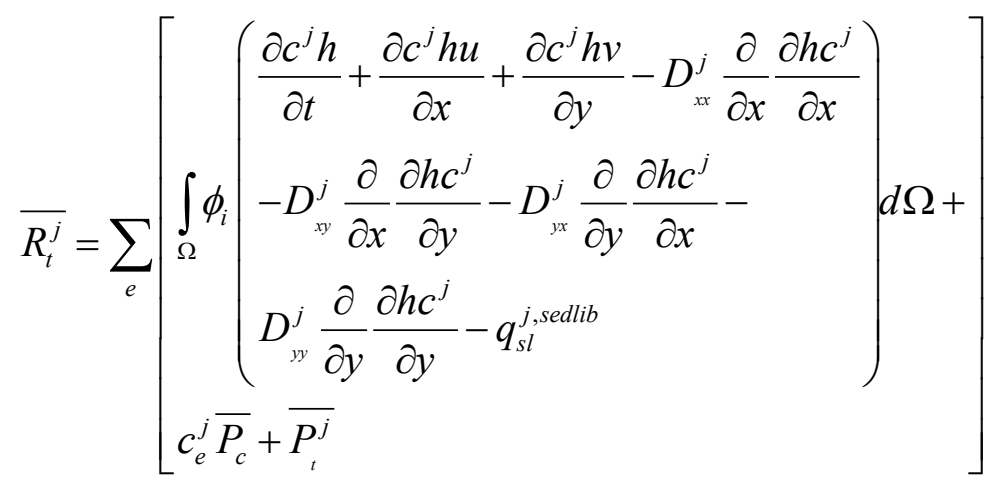

where $j$ is the sediment class, $q_{s l}^{j, s e d l i b}$ is the nodal suspended load normal vertical constituent flux for the $j^{\text {th }}$ grain class supplied by SEDLIB, $\overline{P_{t}^{j}}$ is the SUPG contribution for the $j^{\text {th }}$ grain class, and other variables are as previously defined.

Equation 63 is the sediment suspended load equation. Sediment can also move as bed load, in which case AdH-2D uses a bedload advectiondiffusion equation to compute the bedload transport. This equation can be written in the weak form as the following: 


$$
\left.\overline{R_{b}^{j}}=\sum_{e}\left[\begin{array}{l}
\int_{\Omega} \phi_{i}\left(\begin{array}{l}
\frac{\partial c_{b}^{j} \delta}{\partial t}+\frac{\partial\left(c_{b}^{j} \delta\right) v_{b, x}}{\partial x}+\frac{\partial\left(c_{b}^{j} \delta\right) v_{b, x}}{\partial y}-D_{b, x x}^{j} \frac{\partial}{\partial x} \frac{\partial\left(c_{b}^{j} \delta\right)}{\partial x} \\
-D_{b, x y}^{j} \frac{\partial}{\partial x} \frac{\partial\left(c_{b}^{j} \delta\right)}{\partial y}-D_{b, y x}^{j} \frac{\partial}{\partial y} \frac{\partial\left(c_{b}^{j} \delta\right)}{\partial x} \\
-D_{b, y y}^{j} \frac{\partial}{\partial y} \frac{\partial\left(c_{b}^{j} \delta\right)}{\partial y}-q_{b l}^{j, s e d l i b}-S_{b l}^{j, s e d l i b} \\
P_{b}^{j}
\end{array}\right.
\end{array}\right] d \Omega+\right]
$$

where $\delta$ is the elemental bed layer thickness, $v_{b}$ is the bedload velocity, $c_{b}^{j}$ is the bedload concentration of the $j^{\text {th }}$ class, $D_{b, x x, y y, x y, y x}^{j}$ is the bedload diffusion tensor, $q_{b l}^{j, s e d l i b}$ is the nodal bedload normal vertical constituent flux supplied by SEDLIB, $S_{b l}^{j, \text { sedlib }}$ are the sources/sinks of bedload and $\overline{P_{b}^{j}}$ is the bedload SUPG contribution. The $q_{b l}^{j, s e d i b}$ is computed as follows:

$$
q_{b l}^{j, s e d l i b}=\frac{\left\|v_{b}\right\|}{L_{b}}\left(\beta\left(c^{j} \delta\right)_{e q}-\left(c^{j} \delta\right)\right)
$$

where $\beta$ is the fraction of the $j^{\text {th }}$ class in the bed surface and $L_{b}$ is the bed load adjustment length (given in AdH-2D by the method of Jain). The transient and equilibrium bedload flux are defined as

$$
q_{b l, \text { transient }}^{j, \text { sedlib }}=\left(c_{b}^{j} \delta\right) v_{b}
$$

and

$$
q_{b l, \text { equilibrium }}^{j, \text { slib }}=\beta\left(c_{b}^{j} \delta\right)_{e q} v_{b}
$$

Using Equations 66 and 67, the average bedload flux can be written as

$$
q_{b l}^{j, \text { sedlib }}=\frac{1}{L_{b}}\left(q_{b l, \text { equilibrium }}^{j, \text { sedlib }}-q_{b l, \text { transient }}^{j, \text { sellib }}\right)
$$

Values of the bedload adjustment length, velocities, and equilibrium mass flux are provided by SEDLIB. 
The bedload SUPG term in Equation 64 is

$$
\overline{P_{b}^{j}}=\int_{\Omega} \tau_{b}\left[\nabla\left(v_{b} \phi_{i}\right] \bar{R}_{b}^{j,{ }^{*}} d \Omega\right.
$$

where the strong bedload equation can be written as

$$
\bar{R}_{b}^{j, *}=\frac{\partial\left(c^{j} \delta\right)}{\partial t}+\frac{\partial v_{b, x}\left(c^{j} \delta\right)}{\partial x}+\frac{\partial v_{b, y}\left(c^{j} \delta\right)}{\partial y}-\left(S_{b l}^{j}+q_{b l}^{j, s e d l i b}\right)
$$

The bedload SUPG stabilization coefficient is given as

$$
\tau_{b}=\alpha \frac{l_{e}}{\sqrt{v_{b} \cdot v_{b}}}
$$

where all terms are as previously defined. The bedload diffusion tensor used in AdH-2D is isotropic and is written as

$$
D_{b, x x}^{j}=D_{b, y y}^{j}=D_{b_{x y}}^{j}=D_{b, y x}^{j}=2.5 K \delta u^{*}
$$

where $K$ is a user-defined constant with a default value of 1.0 and $u^{*}$ is the shear velocity provided by SEDLIB. 


\section{Heat Balance and Heat Flux}

AdH-2D has the capability to simulate the exchange of heat between the water column and the atmosphere. This chapter describes in detail the equations used by AdH-2D to compute this heat exchange.

The heat exchange between the atmosphere and the body of water primarily takes place at the air-water interface. The heat exchange is a result of the dynamic interaction between the sources and sinks of heat, as illustrated in Figure 8, and is expressed mathematically by

$$
q_{\text {net }}=q_{s w}+q_{l w}+q_{b}+q_{l}+q_{s e n}+q_{b e d}
$$

where $q_{n e t}$ is the net heat energy stored in the water column, $q_{s w}$ is the short wave radiation, $q_{l w}$ is the long wave radiation, $q_{b}$ is the back radiation, $q_{l}$ is the latent heat of evaporation, $q_{s e n}$ is the sensible heat, and $q_{b e d}$ is the heat transfer between the bed and the water column (usually assumed to be negligible). The heat flux has units of Million Joules per square meter ( $\mathrm{MJ} \mathrm{m}^{-2}$ day $\left.^{-1}\right)$.

Figure 8. Sources and sinks of heat (adapted from Deas and Lowney [2000]).

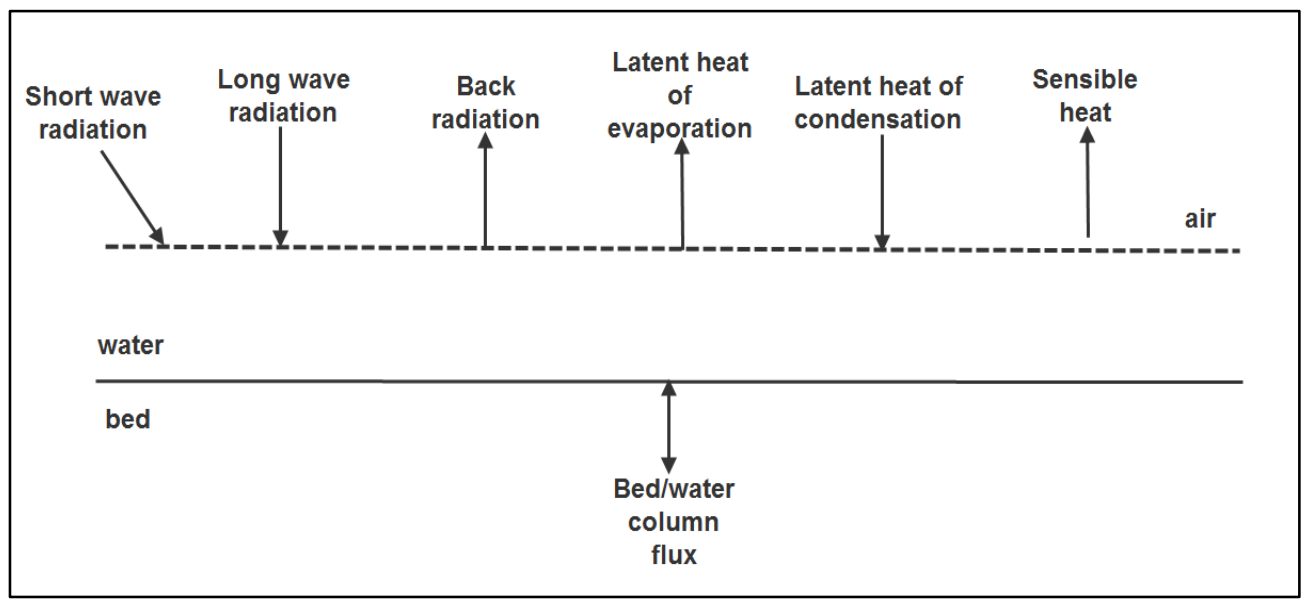

Several methods exist to account for this heat flux between water and air that range from simple, such as the equilibrium temperature method (Edinger et al. 1968), to complex, such as those described in Pond (1975). Conversely, complex methods of heat flux rely on many meteorological parameters to estimate the heat flux at the air-water interface and are in general extremely accurate. However, the data required to correctly apply 
these models are seldom available, and researchers such as Chaudhry et al. (1983) and Ahmad and Sultan (1994), among others, have demonstrated that an equilibrium temperature based model can provide results comparable to those provided by complex models. The following discussion for the simple treatment is taken from Chaudhry et al. (1983) and Ahmad and Sultan (1994).

Equilibrium temperature is a hypothetical/unique water temperature at which the net heat flux at the air-water interface is zero under a set of meteorological conditions. This condition is rarely achieved in the physical world but is very useful in the numerical simulation of heat transfer. Equilibrium temperature is a function of the dew point temperature, the short wave radiation, and the thermal exchange coefficient. The relationship is represented by

$$
T_{e}=T_{d}+\left(\frac{q_{s w}+q_{l w}-q_{l}+q_{s}}{K}\right)
$$

where $T_{e}$ is the equilibrium temperature in degrees Celsius, $T_{d}$ is the dew point temperature in degrees Celsius, and $K$ is the thermal exchange coefficient in $\mathrm{MJ} \mathrm{m}^{-2}$ day $^{-1}{ }^{\circ} \mathrm{C}^{-1}$.

The net heat, $q_{\text {net }}$, is then described by

$$
q_{n e t}=K\left(T_{e}-T_{s}\right)
$$

where $T_{s}$ is the water temperature.

The temperature increase or decrease depends on the net heat flux and is represented by an ordinary differential equation as

$$
\frac{d T_{s}}{d t}=\frac{q_{n e t}}{\left(\rho C_{\rho} H\right)}
$$

where $\rho$ is the fluid density, $C_{\rho}$ is the specific heat of water in Watts (W) Second (sec) per kilogram (Kg) per degree Celsius (C) (W.sec. $\left.\mathrm{Kg}^{-1} \mathrm{C}^{-1}\right)$, and $H$ is water depth. 
In the absence of observed data, the short wave flux can be estimated using

$$
q_{s w}=H_{0} a_{\tau}\left(1-R_{s}\right) C_{a}
$$

where $H_{o}$ is the amount of solar radiation reaching the Earth's outer atmosphere, $a_{\tau}$ is the atmospheric attenuation, and $R_{s}$ is the albedo. The $C_{a}$ is the fraction of solar radiation not absorbed by clouds and can be calculated from

$$
C_{a}=1-0.65 C_{l}^{2}
$$

where $C_{l}$ is the fraction of sky covered by clouds.

The amount of solar radiation reaching the Earth's outer atmosphere is described by

$$
H_{0}=\frac{H_{s c}}{r^{2}}\left\{\sin \phi \sin \delta+\frac{12}{\pi} \cos \phi \cos \delta\left[\sin \left(h_{e}\right)-\sin \left(h_{b}\right]\right\} \Theta\right.
$$

where $H_{s c}$ is the solar constant $\left(1390 \mathrm{Wm}^{-2}\right), r$ is the relative distance between the Earth and the sun, $\phi$ is the local latitude (radians), $\delta$ is the declination (radians), $h_{e}$ is the solar hour angle (radians) at the end of the time period, $h b$ is the solar angle (radians) at the beginning of the time period over which $H_{o}$ is being calculated, and $\Theta$ is a correction factor (o between sunset and sunrise; 1 otherwise). The parameter $r$ is calculated using

$$
r=1.0+0.017 \cos \left[\frac{2 \pi}{365}(185-J D)\right]
$$

where $J D$ is a Julian day (1 on January 1$)$.

The declination, $\delta$, is calculated using

$$
\delta=23.45\left(\frac{2 \pi}{360}\right) \cos \left[\frac{2 \pi}{365}(172-J D)\right]
$$


Hour angles, $h_{b}$ and $h_{e}$, are calculated using

$$
\begin{aligned}
& h_{b}^{*}=\left[\frac{\pi}{12}\left(\left(h_{r}-1\right)-\Delta t_{s}+12\right)\right] \\
& h_{e}^{*}=\left[\frac{\pi}{12}\left(h_{r}-\Delta t_{s}+12\right)\right]
\end{aligned}
$$

for $h_{r} \leq 12$

and

$$
\begin{aligned}
& h_{b}^{*}=\left[\frac{\pi}{12}\left(h_{r}-\Delta t_{s}-12\right)\right] \\
& h_{e}^{*}=\left[\frac{\pi}{12}\left(\left(h_{r}-1\right)-\Delta t_{s}-12\right)\right]
\end{aligned}
$$

for $h_{r}>12$, where $h_{r}$ is the hour of the day (1 to 24 ), and

$$
\begin{aligned}
& h_{b}=h_{b}^{*}-2 \pi \\
& h_{e}=h_{e}^{*}-2 \pi
\end{aligned}
$$

for $h_{b}^{*}>2 \pi, h_{e}^{*}>2 \pi$

$$
\begin{aligned}
& h_{b}=h_{b}^{*}+2 \pi \\
& h_{e}=h_{e}^{*}+2 \pi
\end{aligned}
$$

for $h_{b}^{*}<0, h_{e}^{*}<0$

and

$$
\begin{aligned}
& h_{b}=h_{b}^{*} \\
& h_{e}=h_{e}^{*}
\end{aligned}
$$

for $0 \leq h_{b}^{*} \leq 2 \pi, 0 \leq h_{e}^{*} \leq 2 \pi$ 
The factor $\Delta t_{s}$ is the adjustment for the fraction of the 15-degree increment that the observer is west of the standard meridian for the time zone. This factor is calculated from

$$
\begin{aligned}
& \Delta t_{s}=\frac{-1}{15}\left(L_{s m}-L_{l m}\right) \text { for west longitude } \\
& \Delta t_{s}=\frac{1}{15}\left(L_{s m}-L_{l m}\right) \text { for east longitude }
\end{aligned}
$$

where $L_{s m}$ is the standard meridian (degrees) and $L_{l m}$ is longitude of the local site (degrees).

The long wave radiation is the radiation emitted by the atmosphere and the water and is given by

$$
\begin{aligned}
& q_{l w}=0.97 \sigma\left[\beta\left(T_{a}+273\right)^{4}-\left(T_{s}+273\right)^{4}\right] \\
& \beta=0.74+0.0065 e_{a}\left(1+0.17 C^{2}\right)
\end{aligned}
$$

where $\sigma$ is the Stefan-Boltzmann constant $\left(4.9 \times 10-9 \mathrm{MJ} \mathrm{m}^{-2} \mathrm{~K}^{-4}\right), \beta$ is the atmospheric emissivity, $e_{a}$ is the air-water vapor pressure (millimeter of mercury [mm Hg]), and $C$ is the cloud cover (o for clear and 1 for total cloud cover).

The latent heat of evaporation is given by

$$
q_{l}=0.07 W\left[e_{s}-e_{a}\right]
$$

where $e_{s}$ is the saturated vapor pressure ( $\mathrm{mm} \mathrm{Hg}$ ), $W$ is the wind speed in meters per second, and other variables are as previously defined.

The sensible, or convective, heat flux is expressed as

$$
q_{s}=0.042 \mathrm{~W} \frac{P_{a}}{1000}\left(T_{a}-T_{s}\right)
$$

where $P_{a}$ is the air pressure ( $\left.\mathrm{mm} \mathrm{Hg}\right), T_{a}$ is the air temperature $\left({ }^{\circ} \mathrm{C}\right)$, and other variables are as previously defined. 
If the observed data are not sufficient to compute the full heat balance, AdH-2D uses only the short wave flux to compute heat balance, given as follows:

$$
\begin{gathered}
\frac{d T_{s}}{d t}=\frac{Q_{n e t}}{\rho C_{\rho} H} \\
Q_{n e t}=K_{h}\left(T_{e}-T_{s}\right) \\
T_{e}=T_{d}+\frac{Q_{s}}{K_{h}}
\end{gathered}
$$

where $Q_{n e t}$ and $Q_{s}$ are the net heat flux $\left(\mathrm{Wm}^{-2}\right)$ and short wave flux $\left(\mathrm{Wm}^{-2}\right)$, respectively, $C_{\rho}$ is a function of the water temperature and the wind speed and

$$
\begin{aligned}
& K_{h}=4.5+0.05 T_{s}+(\beta+0.47) f(W) \\
& \beta=0.35+0.015 T_{m}+0.0012 T_{m}^{2} \\
& T_{m}=0.5\left(T_{s}+T_{d}\right) \\
& f(W)=3.3 W
\end{aligned}
$$




\section{Summary}

This report presents the development and implementation of the 2D cartesian and spherical coordinate module of the AdH numerical suite. The report presents a thorough description of the FE method used to solve the $2 \mathrm{D}$ equations, including weak descriptions of the equations, stabilization formulations, and explicitly written integration contributions for the cartesian and spherical equations. 


\section{References}

Ahmad, F., and S. A. R. Sultan. 1994. "Equilibrium Temperature as a Parameter for Estimating the Net Heat flux at the Air-Sea Interface in the Central Red Sea." Oceanologica Acta 17(3): 341-343.

Berger, R. C., and L. M. Lee. 2004. Multidimensional Numerical Modeling of Surges over Initially Dry Land. ERDC/CHL-TR-04-10. Vicksburg, MS: U.S. Army Engineer Research and Development Center.

Bislip-Morales, C., and R. L. Stockstill. 2013. Validation of Modeling Flow Approaching Navigation Locks. ERDC/CHL-TR-13-9. Vicksburg, MS: US Army Engineer Research and Development Center.

Brown, G. L. 2008. "Approximate Profile for Nonequilibrium Suspended Sediment.” Journal of Hydraulic Engineering 134(7).

Carey, G. F., and J. T. Oden. 1986. "Viscous Flows: Velocity-Pressure Form." In Finite Elements: Fluid Mechanics. Vol. VI. The Texas Finite Element Series. Englewood Cliffs, NJ: Prentice-Hall.

Chaudhry, M. H., D. E. Cass, and J. E. Edinger. 1983. "Modeling of Unsteady-Flow Water Temperatures.” Journal of Hydraulic Engineering 109(5): 657-669.

Christensen, B. A. 1972. "Incipient Motion on Cohesionless Channel Banks." Sedimentation, Ch. 4. In Sedimentation: Symposium to honor Professor H.A. Einstein. Edited by H. W. Shen. Fort Collins, CO.

Christensen, B. A. 1995. "Open Channel and Sheet Flow over Flexible Roughness." Proceedings, $21^{\text {st }}$ IAHR Congress, Melbourne, Australia, 19-23 August. Vol.3, $463-467$

Deas, M. L., and C. L. Lowney. 2000. “Water Temperature Modeling Review.” Bay Delta Modeling Forum.

Edinger, J. E., D. W. Duttweller, and J. C. Geyer. 1968. "The Response of Water Temperature to Meteorological Conditions." Water Resources Research 4(5): 1137-1145.

Hendershott, M. C. 1981. "Long Waves and Ocean Tides." Evolution of Physical Oceanography, Ch 10. Cambridge, MA: MIT Press.

Hughes, T. J. R., and A. Brooks. 1982. "Streamline Upwind/Petrov-Galerkin Formulation for Convection Dominated Flows with Particular Emphasis on the Incompressible Navier-Stokes Equations." Computer Methods in Applied Mechanics and Engineering 32(1-3): 199-259.

Jacobs, J. M., and M-H. Wang. 2003. "Atmospheric Momentum Roughness Applied to Stage-Discharge Relationships in Flood Plains.” ASCE Journal of Hydrologic Engineering 8(2): 99-104 
Kelley, C. T., and D. E. Keyes. 1998. "Convergence analysis of Pseudo-Transient Continuation.” SIAM Journal of Numerical Analysis 35(2): 508-523.

Luettich, R. A., and J. J. Westerink. 2004. Formulation and Numerical Development of the 2D/3D ADCIRC Finite Element Model Version 44.XX. https://adcirc.org/files/2018/11/adcirc_theory_2004_12_08.pdf

McAlpin, T. O., R. C. Berger, and A. M. Henville. 2009. Bush Canal Floodgate Study. ERDC/CHL TR-09-9. Vicksburg, MS: US Army Engineer Research and Development Center.

Parker, B. B. 2007. Tidal Analysis and Prediction. National Oceanic and Atmospheric Administration, NOS CO-OPS 3. Silver Spring, MD.

Pond, S. 1975. "The Exchange of Momentum, Heat and Moisture at the OceanAtmosphere Interface.” In Numerical Models of Ocean Circulation: Proceedings of a symposium held at Durham, New Hampshire, October 17-20, 1972. National Academy of Sciences, 26-28.

Pritchard, D. W. 1980. A Summary Concerning the Newly Adopted Practical Salinity, Scale, 1978 and the International Equation of State of Seawater, 1980. New York, NY: Marine Sciences Research Center, State University of New York.

Savant, G., R. C. Berger, T. O. McAlpin, and J. N. Tate. 2011. "Efficient Implicit FiniteElement Hydrodynamic Model for Dam and Levee Breach.” Journal of Hydraulic Engineering 137(9): 1005-1018.

Stockstill, R. L., and R. C. Berger. 1994. HIVEL2D: A Two-Dimensional Flow Model for High-Velocity Channels. Technical Report REMR-HY-12. Vicksburg, MS: US Army Corps of Engineers, US Army Engineer Waterways Experiment Station.

Tate, J. N., R. C. Berger, and R. L. Stockstill. 2006. "Refinement Indicator for Mesh Adaption in Shallow-Water Modeling." Journal of Hydraulic Engineering 132(8): 854-857.

Teeter, A. M. 2002. Sediment Transport in Wind-Exposed Shallow, Vegetated Aquatic Systems. A dissertation. Louisiana State University.

Walters, R., and G. F. Carey. 1984. "Numerical Noise in Ocean and Estuarine Models." Adv. in Water Resources 7(1): 15-20.

Walton R., and B. A. Christensen. 1980. "Friction Factors in Storm Surges over Inland Areas." Journal of the Waterway Port Coastal and Ocean Division 106(2): 261271.

Wu, J. 1982. "Wind-Stress Coefficients over Sea Surface from Breeze to Hurricane." Journal of Geophysical Research 87(C12): 9704-9706. 


\section{Appendix A: Equations of State for Density}

AdH-2D has the capability to transport two kinds of constituents:

(1) barotropic constituents that do not affect the hydrodynamics and

(2) baroclinic constituents that affect the hydrodynamics. For the latter, $\mathrm{AdH}-2 \mathrm{D}$ is formulated to treat density as a linearly varying variable, as described in Equation 52. It can be influenced by salinity, temperature, and sediment. This chapter presents the equations of state used by AdH-2D to compute density from these dependent variables.

\section{Equation of state for the influence of temperature and salinity}

In AdH-2D density is dependent upon salinity and temperature through the equation of state presented in Pritchard (1980). This equation of state is described as follows:

$$
\rho(S, t, 0)=\rho_{w}+\left(b_{0}+b_{1} t+b_{2} t^{2}+b_{3} t^{3}+b_{4} t^{4}\right) S+\left(c_{0}+c_{1} t+c_{2} t^{2}\right) S^{3 / 2}+d_{0} S^{2}
$$

where $\rho(S, t, 0)$ is the density of fluid at a salinity of $S$ (ppt), temperature $t$ $\left({ }^{\circ} \mathrm{C}\right)$, and pressure $\mathrm{o}$ (bars). The variable $\rho_{w}$ is the density of reference pure water, given by

$$
\rho_{w}=a_{0}+a_{1} t+a_{2} t^{2}+a_{3} t^{3}+a_{4} t^{4}+a_{5} t^{5}
$$

The constants in Equations 101 and 102 have the following values:

$$
\begin{aligned}
& a_{o}=999.842594, a_{1}=0.06793952, \text { a2 }=-0.009095290 \\
& a_{3}=0.0001001685, a_{4}=-0.00000120083 \\
& a_{5}=0.000000006536332, b_{o}=0.824493 \\
& b_{1}=-0.0040899, b_{2}=0.000076438 \\
& b_{3}=-0.00000082467, b_{4}=0.0000000053875 \\
& c_{o}=-0.00572466, c_{1}=0.00010277, c_{2}=-0.0000016546 \\
& d_{o}=0.00048315
\end{aligned}
$$




\section{Linearized equation of state for the influence of temperature and salinity}

A linearized form of Equation 96 is available in AdH-2D as well and is expressed as

$$
\rho(S, T, 0)=\rho_{r}+\left[-\alpha\left(T-T_{0}\right)+\beta\left(S-S_{0}\right)\right]
$$

where $\rho_{r}$ is the reference density equal to $1027 \mathrm{~kg} / \mathrm{m}^{3}, T_{o}$ is the reference temperature equal to $10{ }^{\circ} \mathrm{C}$, and $S_{o}$ is the reference salinity equal to $35 \mathrm{PSU}^{*}$. The constant $\alpha$ is the coefficient of thermal expansion and is equal to $0.15 \mathrm{~kg} /\left(\mathrm{m}^{3} .{ }^{\circ} \mathrm{C}\right),{ }^{\dagger}$ and $\beta$ is the coefficient of saline contraction and is equal to $0.78 \mathrm{~kg} /\left(\mathrm{m}^{3}\right.$.parts per thousand [ppt]).

\section{Equation of state for the influence of sediments}

AdH-2D has the capability to transport sediment in suspension as well as in bedload. The presence of sediment may have an influence on the density of the fluid. This relationship is presented in $\mathrm{AdH}$ as

$$
\rho(S, T, 0, C)=\rho(S, T, 0)+\Delta \rho
$$

where $\Delta \rho$ is the change in density due to sediment, $C$ (kilogram/kilogram) is the total concentration of sediment in the water column, and other variables are as previously defined. The density change, $\Delta \rho$, is expressed as follows:

$$
\Delta \rho=\sum_{i=1}^{n g} C_{i}\left(1-S G_{i}\right) /\left[S G_{i}-C_{i}\left(S G_{i}-1\right)\right]
$$

where $n g$ is the number of sediment classes, $C i$ is concentration of the $i^{\text {th }}$ sediment class, and $S G_{i}$ is the specific gravity of the $i^{\text {th }}$ sediment class.

\footnotetext{
* Practical Salinity Unit

$\dagger \mathrm{kg}=$ kilogram; $m$ = meter
} 


\section{Appendix B: Discretization and Integration of Individual Terms}

This appendix presents the detailed breakdown of the integrations for all the terms composing the weak continuity and momentum equations.

Figure 9 lists the AdH-2D routines where these integrations are performed.

Figure 9. AdH-2D integration routines.

\begin{tabular}{cc|}
\hline Integration & AdH-2D Routine \\
\hline Temporal terms & fe_sw2_2d_elem_resid.c \\
& fe_1v2d_uupc.c \\
\hline Convection terms & fe_sw2_convection.c \\
& fe_sw2_1d_convection.c \\
\hline Pressure terms & fe_sw2_pressure.c \\
& fe_sw2_1d_pressure.c \\
\hline Bodyforce terms & fe_sw2_bodyforce.c \\
\hline Reynolds stress terms & fe_sw2_2d_elem_resid.c \\
& fe_sw2_stodpai.c \\
\hline Friction terms & fe_sw2_2d_friction.c \\
& fe_sw2_1d_friction.c \\
\hline Coriolis terms & fe_sw2_coriolis \\
\hline Rainfall and/or evaporation & fe_sw2_2d_elem_resid \\
terms & fe_sw2_surface_stress.c \\
\hline Wind and/or wave stress & \\
terms & fe_sw2_wd_pg.c \\
\hline Petrov-Galerkin terms & \\
\hline
\end{tabular}

\section{Continuity equation}

This section describes the FE discretization of the continuity equation.

\section{Temporal term}

The temporal term $\frac{\partial h}{\partial t}$ is integrated consistently as follows: 


$$
\frac{1}{\Delta t} \int_{\Omega} \phi_{i} h^{k} d \Omega=\frac{1}{\Delta t} \int_{\xi=0}^{1} \int_{\eta=0}^{1-\xi} \phi_{i}\left(\phi_{1} h_{1}^{k}+\phi_{2} h_{2}^{k}+\phi_{3} h_{3}^{k}\right)(2 \Lambda) d \eta d \xi
$$

where

$k=$ the time under consideration $(\mathrm{t}-1, \mathrm{t}$, or $\mathrm{t}+1)$.

\section{Convective storage/change term}

The $\frac{\partial h u}{\partial x}$ and $\frac{\partial h v}{\partial y}$ terms form the convective storage terms in the continuity equation. The weak form of these terms is integrated using integration by parts as follows:

$$
\begin{aligned}
& \int_{\Omega} \frac{\partial}{\partial x}(h u) \phi_{i} d \Omega=-\int_{\Omega}(h u) \frac{\partial \phi_{i}}{\partial x} d \Omega+\int_{\Gamma}(h u) \phi_{i} \mathbf{g}_{x} d \Gamma \\
& \int_{\Omega} \frac{\partial}{\partial y}(h v) \phi_{i} d \Omega=-\int_{\Omega}(h v) \frac{\partial \phi_{i}}{\partial y} d \Omega+\int_{\Gamma}(h v) \phi_{i} \mathrm{~g}_{y} d \Gamma
\end{aligned}
$$

The second term on the right-hand side (RHS) in Equations 102 and 103 is the line integral over the element boundary multiplied by the shape function. These terms will be discussed later in this appendix. These integrals can be simplified for linear basis functions on triangles to

$x$-direction terms:

$$
\begin{gathered}
-\int_{\Omega}(h u) \frac{\partial \phi_{i}}{\partial x} d \Omega=-\frac{\partial \phi_{i}}{\partial x} \int_{\Omega}(h)(u) d \Omega=-\frac{\partial \phi_{i}}{\partial x} \int_{\xi=0}^{1} \int_{\eta=0}^{1-\xi}(h)(u)(2 \Lambda) d \eta d \xi \\
-\int_{\Omega}(h u) \frac{\partial \phi_{i}}{\partial x} d \Omega=-\frac{\partial \phi_{i}}{\partial x} \int_{\xi=0}^{1} \int_{\eta=0}^{1-\xi}(h)(u)(2 \Lambda) d \eta d \xi
\end{gathered}
$$

$y$-direction terms:

$$
-\int_{\Omega}(h v) \frac{\partial \phi_{i}}{\partial y} d \Omega=-\frac{\partial \phi_{i}}{\partial y} \int_{\Omega}(h)(v) d \Omega=-\frac{\partial \phi_{i}}{\partial y} \int_{\xi=0}^{1} \int_{\eta=0}^{1-\xi}(h)(v)(2 \Lambda) d \eta d \xi
$$




$$
-\int_{\Omega}(h v) \frac{\partial \phi_{i}}{\partial y} d \Omega=-\frac{\partial \phi_{i}}{\partial y} \int_{\xi=0}^{1} \int_{\eta=0}^{1-\xi}(h)(v)(2 \Lambda) d \eta d \xi
$$

\section{Momentum equations}

This section describes the discretization of the $x$-direction and the $y$-direction momentum equations.

\section{Temporal term}

The temporal terms ( $\frac{\partial h u}{\partial t}$ and $\left.\frac{\partial h v}{\partial x}\right)$ can be integrated either consistently or in a lumped fashion. For stability purposes, AdH-2D uses the lumped integration technique, though both integrations are provided below for completeness.

x-direction term: Consistent integration

$$
\frac{1}{\Delta t} \int_{\Omega} \phi_{i}(h u)^{k} d \Omega=\frac{1}{\Delta t} \int_{\xi=0}^{1} \int_{\eta=0}^{1-\xi} \phi_{i}\left(\phi_{1} h_{1}^{k} u_{1}^{k}+\phi_{2} h_{2}^{k} u_{2}^{k}+\phi_{3} h_{3}^{k} u_{3}^{k}\right)(2 \Lambda) d \eta d \xi
$$

$x$-direction term: Lumped integration

$$
\begin{gathered}
\frac{1}{\Delta t} \int_{\Omega} \phi_{i}(h u) d \Omega=\frac{1}{\Delta t} \int_{\xi=0}^{1} \int_{\eta=0}^{1-\xi}(h u)(2 \Lambda) d \eta d \xi \\
\frac{1}{\Delta t} \int_{\Omega} \phi_{i}(h u) d \Omega=\frac{1}{3} \frac{2 \Lambda}{\Delta t}(h u)_{i}
\end{gathered}
$$

$y$-direction term: Consistent integration

$$
\frac{1}{\Delta t} \int_{\Omega} \phi_{i}(h v)^{k} d \Omega=\frac{1}{\Delta t} \int_{\xi=0}^{1} \int_{\eta=0}^{1-\xi} \phi_{i}\left(\phi_{1} h_{1}^{k} v_{1}^{k}+\phi_{2} h_{2}^{k} v_{2}^{k}+\phi_{3} h_{3}^{k} v_{3}^{k}\right)(2 \Lambda) d \eta d \xi
$$

$y$-direction term: Lumped integration

$$
\frac{1}{\Delta t} \int_{\Omega} \phi_{i} \partial(h v) d \Omega=\frac{1}{\Delta t} \int_{\xi=0}^{1} \int_{\eta=0}^{1-\xi}(h v)(2 \Lambda) d \eta d \xi
$$




$$
\frac{1}{\Delta t} \int_{\Omega} \phi_{i} \partial(h v) d \Omega=\frac{1}{3} \frac{2 \Lambda}{\Delta t}(h v)_{i}
$$

where all variables are as previously defined.

\section{Convective terms}

The $\frac{\partial h u u}{\partial x}, \frac{\partial h v u}{\partial y}, \frac{\partial h v u}{\partial x}$, and $\frac{\partial h v v}{\partial y}$ terms $i$ form the convection/advection terms in the $x$ - and $y$-direction momentum equations. This section will detail the FE discretization of each of these terms.

The weak form of these terms is integrated by parts as follows:

$$
\begin{aligned}
& \int_{\Omega} \frac{\partial}{\partial x}(h u u) \phi_{i} d \Omega=-\int_{\Omega}(h u u) \frac{\partial \phi_{i}}{\partial x} d \Omega+\int_{\Gamma}(h u u) \phi_{i} g_{x} d \Gamma \\
& \int_{\Omega} \frac{\partial}{\partial y}(h u v) \phi_{i} d \Omega=-\int_{\Omega}(h u v) \frac{\partial \phi_{i}}{\partial y} d \Omega+\int_{\Gamma}(h u v) \phi_{i} g_{y} d \Gamma \\
& \int_{\Omega} \frac{\partial}{\partial x}(h u v) \phi_{i} d \Omega=-\int_{\Omega}(h u v) \frac{\partial \phi_{i}}{\partial x} d \Omega+\int_{\Gamma}(h u v) \phi_{i} g_{x} d \Gamma \\
& \int_{\Omega} \frac{\partial}{\partial y}(h v v) w_{i} d \Omega=-\int_{\Omega}(h v v) \frac{\partial w_{i}}{\partial y} d \Omega+\int_{\Gamma}(h v v) \phi_{i} g_{y} d \Gamma
\end{aligned}
$$

Again, the second terms on the RHSs are the line integrals over the element boundary multiplied by the shape function. The first term on the RHS in Equation 114 is integrated for linear triangles as follows:

$$
\begin{array}{r}
-\int_{\Omega}(h u u) \frac{\partial \phi_{i}}{\partial x} d \Omega=-\frac{\partial \phi_{i}}{\partial x} \int_{\Omega}(h)(u)(u) d \Omega=-\frac{\partial \phi_{i}}{\partial x} \int_{\xi=0}^{1} \int_{\eta=0}^{1-\xi}(h)(u)(u)(2 \Lambda) d \eta d \xi \\
=-\frac{\partial \phi_{i}}{\partial x} \frac{\Lambda}{30}\left[\begin{array}{l}
u_{1}^{2}\left(3 h_{1}+h_{2}+h_{3}\right)+u_{2}^{2}\left(h_{1}+3 h_{2}+h_{3}\right)+ \\
u_{3}^{2}\left(h_{1}+h_{2}+3 h_{3}\right)+ \\
u_{1} u_{2}\left(2 h_{1}+2 h_{2}+h_{3}\right)+u_{1} u_{3}\left(2 h_{1}+h_{2}+2 h_{3}\right)+ \\
u_{2} u_{3}\left(h_{1}+2 h_{2}+2 h_{3}\right)
\end{array}\right]
\end{array}
$$


The first term on the RHS in Equation 115 is integrated as follows:

$$
\left.\begin{array}{rl}
-\int_{\Omega}(h u v) \frac{\partial \phi_{i}}{\partial y} d \Omega=-\frac{\partial \phi_{i}}{\partial y} \int_{\Omega}(h)(u)(v) d \Omega=-\frac{\partial \phi_{i}}{\partial y} \int_{\xi=0}^{1} \int_{\eta=0}^{1-\xi}(h)(u)(v)(2 \Lambda) d \eta d \xi \\
=-\frac{\partial \phi_{i}}{\partial y} \frac{\Lambda}{60}\left\{\begin{array}{l}
h_{2}\left[\begin{array}{l}
u_{1}\left(6 v_{1}+2 v_{2}+2 v_{3}\right)+u_{2}\left(2 v_{1}+2 v_{2}+v_{3}\right)+ \\
u_{3}\left(2 v_{1}+v_{2}+2 v_{3}\right)
\end{array}\right] \\
\left.+\begin{array}{l}
u_{1}\left(2 v_{1}+2 v_{2}+v_{3}\right)+u_{2}\left(2 v_{1}+6 v_{2}+2 v_{3}\right)+ \\
u_{3}\left(v_{1}+2 v_{2}+2 v_{3}\right)
\end{array}\right] \\
+\left[\begin{array}{l}
u_{1}\left(2 v_{1}+v_{2}+2 v_{3}\right)+u_{2}\left(v_{1}+2 v_{2}+2 v_{3}\right)+ \\
u_{3}\left(2 v_{1}+2 v_{2}+6 v_{3}\right)
\end{array}\right]
\end{array}\right]
\end{array}\right\}
$$

The first term on the RHS in Equation 116 is integrated as follows:

$$
\left.\begin{array}{rl}
-\int_{\Omega}(h u v) \frac{\partial \phi_{i}}{\partial x} d \Omega=-\frac{\partial \phi_{i}}{\partial x} \int_{\Omega}(h)(u)(v) d \Omega=-\frac{\partial \phi_{i}}{\partial x} \int_{\xi=0}^{1} \int_{\eta=0}^{1-\xi}(h)(u)(v)(2 \Lambda) d \eta d \xi \\
=-\frac{\partial \phi_{i}}{\partial x} \frac{\Lambda}{60}\left\{\begin{array}{l}
h_{2}\left[\begin{array}{l}
\left.u_{1}\left(6 v_{1}+2 v_{2}+2 v_{3}\right)+u_{2}\left(2 v_{1}+2 v_{2}+v_{3}\right)+\right] \\
u_{3}\left(2 v_{1}+v_{2}+2 v_{3}\right)
\end{array}\right] \\
h_{2}\left(2 v_{1}+2 v_{2}+v_{3}\right)+u_{2}\left(2 v_{1}+6 v_{2}+2 v_{3}\right)+
\end{array}\right] \\
\left.+\begin{array}{l}
\left.u_{2}+2 v_{3}\right) \\
{\left[\begin{array}{l}
u_{1}\left(2 v_{1}+v_{2}+2 v_{3}\right)+u_{2}\left(v_{1}+2 v_{2}+2 v_{3}\right)+ \\
u_{3}\left(2 v_{1}+2 v_{2}+6 v_{3}\right)
\end{array}\right]}
\end{array}\right]
\end{array}\right\}
$$


The first term on the RHS in Equation 117 is integrated as follows:

$$
\begin{array}{r}
-\int_{\Omega}(h v v) \frac{\partial \phi_{i}}{\partial y} d \Omega=-\frac{\partial \phi_{i}}{\partial y} \int_{\Omega}(h)(v)(v) d \Omega=-\frac{\partial \phi_{i}}{\partial y} \int_{\xi=0}^{1} \int_{\eta=0}^{1-\xi}(h)(v)(v)(2 \Lambda) d \eta d \xi \\
=-\frac{\partial \phi_{i}}{\partial y} \frac{\Lambda}{30}\left[\begin{array}{l}
v_{1}^{2}\left(3 h_{1}+h_{2}+h_{3}\right)+v_{2}^{2}\left(h_{1}+3 h_{2}+h_{3}\right)+ \\
v_{3}^{2}\left(h_{1}+h_{2}+3 h_{3}\right)+ \\
v_{1} v_{2}\left(2 h_{1}+2 h_{2}+h_{3}\right)+v_{1} v_{3}\left(2 h_{1}+h_{2}+2 h_{3}\right)+ \\
v_{2} v_{3}\left(h_{1}+2 h_{2}+2 h_{3}\right)
\end{array}\right]
\end{array}
$$

\section{Pressure terms}

The terms $\frac{\partial}{\partial x}\left(\frac{1}{2} \frac{\rho}{\rho_{0}} g h^{2}\right)$ and $\frac{\partial}{\partial y}\left(\frac{1}{2} \frac{\rho}{\rho_{0}} g h^{2}\right)$ represent the pressure terms in the $x$ - and $y$-direction momentum equations. Multiplying with the weight function and integrating by parts results in the following:

$$
\int_{\Omega} \frac{\partial}{\partial x}\left(\frac{1}{2} \frac{\rho}{\rho_{0}} g h^{2}\right) \phi_{i} d \Omega=-\int_{\Omega}^{1} \frac{\rho}{2} \frac{\rho}{\rho_{0}} g h^{2} \frac{\partial \phi_{i}}{\partial x} d \Omega+\int_{\Gamma} \frac{1}{2} \frac{\rho}{\rho_{0}} g h^{2} \phi_{i} \mathrm{~g}_{x} d \Gamma
$$

and

$$
\int_{\Omega} \frac{\partial}{\partial y}\left(\frac{1}{2} \frac{\rho}{\rho_{0}} g h^{2}\right) \phi_{i} \partial \Omega=-\int_{\Omega} \frac{1}{2} \frac{\rho}{\rho_{0}} g h^{2} \frac{\partial \phi_{i}}{\partial y} d \Omega+\int_{\Gamma} \frac{1}{2} \frac{\rho}{\rho_{0}} g h^{2} \phi_{i} g_{y} d \Gamma
$$

The second terms on each RHS are the line integrals over the element boundary multiplied by the shape function. These terms are used in AdH-2D to determine the model domain and are explained later in this report.

For a constant density, the first terms in Equations 122 and 123 are integrated as follows:

$$
\begin{aligned}
-\int_{\Omega} \frac{1}{2} \frac{\rho}{\rho_{0}} g h^{2} \frac{\partial \phi_{i}}{\partial x} d \Omega & =-\frac{1}{2} \frac{\rho}{\rho_{0}} g \frac{\partial \phi_{i}}{\partial x} \int_{\Omega} h^{2} d \Omega \\
& =-\frac{1}{12} \frac{\rho}{\rho_{0}} g \Lambda \frac{\partial \phi_{i}}{\partial x}\left(h_{1}^{2}+h_{2}^{2}+h_{3}^{2}+h_{1} h_{2}+h_{1} h_{3}+h_{2} h_{3}\right)
\end{aligned}
$$


and

$$
\begin{aligned}
-\int_{\Omega} \frac{1}{2} \frac{\rho}{\rho_{0}} g h^{2} \frac{\partial \phi_{i}}{\partial y} d \Omega & =-\frac{1}{2} \frac{\rho}{\rho_{0}} g \frac{\partial \phi_{i}}{\partial y} \int_{\Omega} h^{2} d \Omega \\
& =-\frac{1}{12} \frac{\rho}{\rho_{0}} g \Lambda \frac{\partial \phi_{i}}{\partial y}\left(h_{1}^{2}+h_{2}^{2}+h_{3}^{2}+h_{1} h_{2}+h_{1} h_{3}+h_{2} h_{3}\right)
\end{aligned}
$$

If density is treated as a linearly varying variable, however, so that

$$
\rho=\sum_{0}^{3} \rho_{i} \phi_{i}=\rho_{1} \phi_{1}+\rho_{2} \phi_{2}+\rho_{3} \phi_{3}
$$

Then, Equations 122 and 123 are integrated as follows:

$$
\begin{aligned}
-\int_{\Omega} \frac{1}{2} \frac{\rho}{\rho_{0}} g h^{2} \frac{\partial \phi_{i}}{\partial x} d \Omega & =-\frac{g}{2 \rho_{0}} \frac{\partial \phi_{i}}{\partial x} \int_{\Omega} \rho h^{2} d \Omega \\
& =-\frac{g}{2 \rho_{0}} \frac{\partial \phi_{i}}{\partial x} \int_{\xi=0}^{1} \int_{\eta=0}^{1-\xi}(\rho)\left(h^{2}\right)(2 \Lambda) d \eta d \xi \\
& =-\frac{g \Lambda}{30 \rho_{0}} \frac{\partial \phi_{i}}{\partial x}\left[\begin{array}{l}
h_{1}^{2}\left(3 \rho_{1}+\rho_{2}+\rho_{3}\right)+ \\
h_{2}^{2}\left(\rho_{1}+3 \rho_{2}+\rho_{3}\right)+ \\
h_{3}^{2}\left(\rho_{1}+\rho_{2}+3 \rho_{3}\right)+ \\
h_{1} h_{2}\left(2 \rho_{1}+2 \rho_{2}+\rho_{3}\right)+ \\
h_{1} h_{3}\left(2 \rho_{1}+\rho_{2}+2 \rho_{3}\right)+ \\
h_{2} h_{3}\left(\rho_{1}+2 \rho_{2}+2 \rho_{3}\right)
\end{array}\right]
\end{aligned}
$$


and

$$
\begin{aligned}
-\int_{\Omega} \frac{1}{2} \frac{\rho}{\rho_{0}} g h^{2} \frac{\partial \phi_{i}}{\partial y} d \Omega & =-\frac{g}{2 \rho_{0}} \frac{\partial \phi_{i}}{\partial y} \int_{\Omega} \rho h^{2} d \Omega \\
& =-\frac{g}{2 \rho_{0}} \frac{\partial \phi_{i}}{\partial y} \int_{\xi=0}^{1} \int_{\eta=0}^{1-\xi}(\rho)\left(h^{2}\right)(2 \Lambda) d \eta d \xi \\
& =-\frac{g \Lambda}{30 \rho_{0}} \frac{\partial \phi_{i}}{\partial y}\left[\begin{array}{l}
h_{1}^{2}\left(3 \rho_{1}+\rho_{2}+\rho_{3}\right)+ \\
h_{2}^{2}\left(\rho_{1}+3 \rho_{2}+\rho_{3}\right)+ \\
h_{3}^{2}\left(\rho_{1}+\rho_{2}+3 \rho_{3}\right)+ \\
h_{1} h_{2}\left(2 \rho_{1}+2 \rho_{2}+\rho_{3}\right)+ \\
h_{1} h_{3}\left(2 \rho_{1}+\rho_{2}+2 \rho_{3}\right)+ \\
h_{2} h_{3}\left(\rho_{1}+2 \rho_{2}+2 \rho_{3}\right)
\end{array}\right]
\end{aligned}
$$

\section{Reynolds stress terms}

The Reynolds stress contributions to the momentum terms $-\frac{\partial}{\partial x}\left(\frac{h}{\rho_{0}} \sigma_{x x}\right)$,

$-\frac{\partial}{\partial y}\left(\frac{h}{\rho_{0}} \sigma_{y x}\right),-\frac{\partial}{\partial x}\left(\frac{h}{\rho_{0}} \sigma_{x y}\right)$, and $-\frac{\partial}{\partial y}\left(\frac{h}{\rho_{0}} \sigma_{y y}\right)$, where $\sigma_{x x}, \sigma_{x y}, \sigma_{y y}$, and

$\sigma_{y x}$ are Reynolds stresses computed outside the FE framework using

Equations 6, 7, 8, and 9. For these terms, AdH uses elemental averages for depth and Reynolds stresses for stability. Multiplying by the weight function and integrating by parts results in the following:

$$
\begin{aligned}
& -\int_{\Omega} \frac{\partial}{\partial x}\left(\sigma_{x x} \frac{h_{e}}{\rho_{0}}\right) \phi_{i} \partial \Omega=\int_{\Omega} \sigma_{x x} \frac{h_{e}}{\rho_{0}} \frac{\partial \phi_{i}}{\partial x} d \Omega-\int_{\Gamma} \sigma_{x x} \frac{h_{e}}{\rho_{0}} \phi_{i} g g_{x} d \Gamma \\
& -\int_{\Omega} \frac{\partial}{\partial y}\left(\sigma_{x y} \frac{h_{e}}{\rho_{0}}\right) \phi_{i} \partial \Omega=\int_{\Omega} \sigma_{x y} \frac{h_{e}}{\rho_{0}} \frac{\partial \phi_{i}}{\partial y} d \Omega-\int_{\Gamma} \sigma_{x y} \frac{h_{e}}{\rho_{0}} \phi_{i} g_{y} d \Gamma \\
& -\int_{\Omega} \frac{\partial}{\partial x}\left(\sigma_{y x} \frac{h_{e}}{\rho_{0}}\right) \phi_{i} \partial \Omega=\int_{\Omega} \sigma_{y x} \frac{h_{e}}{\rho_{0}} \frac{\partial \phi_{i}}{\partial x} d \Omega-\int_{\Gamma} \sigma_{y x} \frac{h_{e}}{\rho_{0}} \phi_{i} g \eta_{x} d \Gamma \\
& -\int_{\Omega} \frac{\partial}{\partial y}\left(\sigma_{y y} \frac{h_{e}}{\rho_{0}}\right) \phi_{i} \partial \Omega=\int_{\Omega} \sigma_{y y} \frac{h_{e}}{\rho_{0}} \frac{\partial \phi_{i}}{\partial y} d \Omega-\int_{\Gamma} \sigma_{y y} \frac{h_{e}}{\rho_{0}} \phi_{i} g g_{y} d \Gamma
\end{aligned}
$$


where $h_{e}$ is the elementally averaged depth. AdH-2D assumes that there are no Reynolds stress contributions from the boundary.

The first term on the RHS of these equations can be simplified on linear triangles with elementally averaged depths and stresses to give

$$
\begin{aligned}
\int_{\Omega} \sigma_{x x} \frac{h_{e}}{\rho_{0}} \frac{\partial \phi_{i}}{\partial x} d \Omega & =\frac{\sigma_{x x} h_{e}}{\rho_{0}} \frac{\partial \phi_{i}}{\partial x} \int_{\Omega} d \Omega \\
& =2 \Lambda \frac{\sigma_{x x} h_{e}}{\rho_{0}} \frac{\partial \phi_{i}}{\partial x} \\
\int_{\Omega} \sigma_{x y} \frac{h_{e}}{\rho_{0}} \frac{\partial \phi_{i}}{\partial y} d \Omega & =\frac{\sigma_{x y} h_{e}}{\rho_{0}} \frac{\partial \phi_{i}}{\partial y} \int_{\Omega} d \Omega \\
& =2 \Lambda \frac{\sigma_{x y} h_{e}}{\rho_{0}} \frac{\partial \phi_{i}}{\partial y} \\
\int_{\Omega} \sigma_{y x} \frac{h_{e}}{\rho_{0}} \frac{\partial \phi_{i}}{\partial x} d \Omega & =\frac{\sigma_{y x} h_{e}}{\rho_{0}} \frac{\partial \phi_{i}}{\partial x} \int_{\Omega} d \Omega \\
& =2 \Lambda \frac{\sigma_{y x}}{\rho_{0}} \frac{\partial \phi_{i}}{\partial x} \\
\int_{\Omega} \sigma_{y y} \frac{h_{e}}{\rho_{0}} \frac{\partial \phi_{i}}{\partial y} d \Omega & =\frac{\sigma_{y y} h_{e}}{\rho_{0}} \frac{\partial \phi_{i}}{\partial y} \int_{\Omega} d \Omega \\
& =2 \Lambda \frac{\sigma_{y y} h_{e}}{\rho_{0}} \frac{\partial \phi_{i}}{\partial y}
\end{aligned}
$$

AdH-2d assumes that boundary contributions from Reynolds stress terms are zero (second term on the RHSs of equations 128-131).

\section{Body force terms}

Gravity body force terms

The momentum contributions, $\frac{\rho}{\rho_{0}} g h \frac{\partial z_{b}}{\partial x}$ and $\frac{\rho}{\rho_{0}} g h \frac{\partial z_{b}}{\partial y}$ are gravitational body force terms. These contributions are multiplied by the weight function and integrated consistently to obtain 


$$
\int_{\Omega} \frac{\rho}{\rho_{0}} g h \frac{\partial z}{\partial x} \phi_{i} d \Omega=\frac{\rho}{\rho_{0}} \int_{\xi=0}^{1} \int_{\eta=0}^{1-\xi} \frac{\partial z}{\partial x} \phi_{i}\left(\phi_{0} h_{0}+\phi_{1} h_{1}+\phi_{2} h_{2}\right) 2 \Lambda d \eta d \xi
$$

and

$$
\int_{\Omega} \frac{\rho}{\rho_{0}} g h \frac{\partial z}{\partial y} \phi_{i} d \Omega=\frac{\rho}{\rho_{0}} \int_{\xi=0}^{1} \int_{\eta=0}^{1-\xi} \frac{\partial z}{\partial y} \phi_{i}\left(\phi_{0} h_{0}+\phi_{1} h_{1}+\phi_{2} h_{2}\right) 2 \Lambda d \eta d \xi
$$

Friction stress body force terms

The terms $\frac{C_{f} u\|V\|}{2}$ and $\frac{C_{f} v\|V\|}{2}$ are the friction stress terms in the $x$ - and $y$-direction momentum equations, respectively. These terms are multiplied with the weight function and integrated consistently to obtain

$$
\int_{\Omega} \frac{C_{f} u\|V\|}{2} \phi_{i} d \Omega=\int_{\xi=0}^{1} \int_{\eta=0}^{1-\xi} \frac{C_{f}}{2}\|V\| \phi_{i}\left(\phi_{0} u_{0}+\phi_{1} u_{1}+\phi_{2} u_{2}\right) 2 \Lambda d \eta d \xi
$$

and

$$
\int_{\Omega} \frac{C_{f} v\|V\|}{2} \phi_{i} d \Omega=\int_{\xi=0}^{1} \int_{\eta=0}^{1-\xi} \frac{C_{f}}{2}\|V\| \phi_{i}\left(\phi_{0} v_{0}+\phi_{1} v_{1}+\phi_{2} v_{2}\right) 2 \Lambda d \eta d \xi
$$

where $\|V\|$ is the elementally averaged velocity magnitude.

\section{Coriolis force terms}

The $f v h$ and $f u h$ terms in the $x$ - and $y$-direction momentum equations are the Coriolis force terms. These terms are multiplied by the weight function and consistently integrated to obtain the following:

$$
\int_{\Omega}(f v h) \phi_{i} d \Omega=\int_{\xi=0}^{1} \int_{\eta=0}^{1-\xi} \phi_{i}(v)(h) 2 \lambda \sin (\varphi) 2 \Lambda d \eta d \xi
$$

and

$$
\int_{\Omega}(f u h) \phi_{i} d \Omega=\int_{\xi=0}^{1} \int_{\eta=0}^{1-\xi} \phi_{i}(u)(h) 2 \lambda \sin (\varphi) 2 \Lambda d \eta d \xi
$$


where $\varphi$ the latitude, and $\lambda$ is the angular velocity of Earth.

All elemental/body integrations for each 2D equation have been given. The next section describes the FE integration of the boundary terms (line integrals) that arise from the integration by parts of the weak contributions to both the depth-averaged continuity and momentum equations.

\section{Boundary line integration terms}

Continuity convective storage/change terms

The terms $\int_{\Gamma}(h u) \phi_{i} g_{x} d \Gamma$ and $\int_{\Gamma}(h v) \phi_{i} \mathrm{~g}_{y} d \Gamma$ are the boundary line integration in equations 102 and 103, respectively. These terms are integrated based on $1 \mathrm{D}$ basis functions defined as

$$
\begin{aligned}
& \phi_{1 e, 1}=1-\xi \\
& \phi_{1 e, 2}=\xi
\end{aligned}
$$

where the subscript " $1 e$ " denotes one dimensional (1D) expansions over a $1 \mathrm{D}$ element.

The integration of these terms involves 1D elements as shown in Figure 10.

Figure 10. 1D Line integral basis functions.

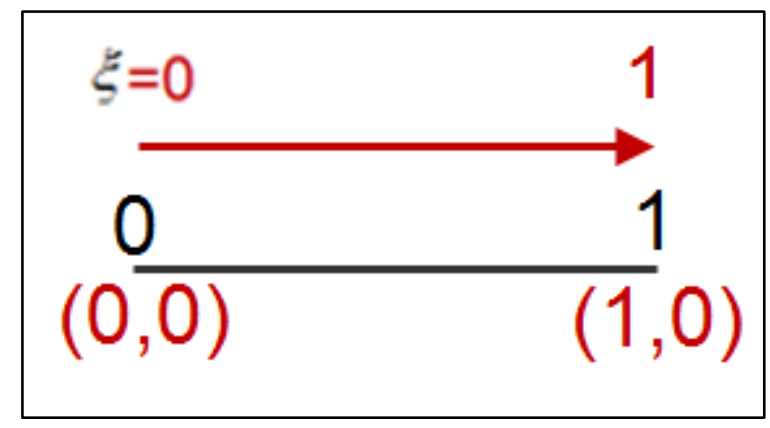

The basis function definitions in Equation 142 are used to expand the variables as

$$
u_{1 e}=\sum_{1}^{2} u_{1 e, i} \phi_{1 e, i}(x, y)=u_{1 e, 1} \phi_{1 e, 1}(x, y)+u_{1 e, 2} \phi_{1 e, 2}(x, y)
$$




$$
\begin{aligned}
& v_{1 e}=\sum_{1}^{2} v_{1 e, i} \phi_{1 e, i}(x, y)=v_{1 e, 1} \phi_{1 e, 1}(x, y)+v_{1 e, 2} \phi_{1 e, 2}(x, y) \\
& h_{1 e}=\sum_{1}^{2} h_{1 e, i} \phi_{1 e, i}(x, y)=h_{1 e, 1} \phi_{1 e, 1}(x, y)+h_{1 e, 2} \phi_{1 e, 2}(x, y)
\end{aligned}
$$

where the variables are as previously defined.

Applying this gives

$$
\begin{gathered}
\int_{\Gamma}(h u)_{1 e} \phi_{1 e, i} g_{x} d \Gamma=n_{x} \int_{\xi=0}^{1} \phi_{1 e, i}(h u)_{1 e} L d \xi \\
=\Delta y \int_{\xi=0}^{1} \phi_{1 e, i}(h u)_{1 e} L d \xi
\end{gathered}
$$

where $n_{x}$ is the normal in the $x$-direction for the line segment and $\Delta y$ is $y$-ordinate distance between the nodes on the line segment. For a $1 \mathrm{D}$ element, $n_{x}$ is equal to the $y$-ordinate distance between the nodes on the element.

The second integral gives

$$
\begin{gathered}
\int_{\Gamma}(h v)_{1 e} \phi_{1 e, i} g_{y} d \Gamma=n_{y} \int_{\xi=0}^{1} \phi_{1 e, i}(h v)_{1 e} L d \xi \\
=-\Delta x \int_{\xi=0}^{1} \phi_{1 e, i}(h v)_{1 e} L d \xi
\end{gathered}
$$

where $n_{y}$ is the normal in the $y$-direction for the line segment and $L$ is the length of the line segment. For a $1 \mathrm{D}$ element, $n_{y}$ is equal to $-\Delta x$, where $\Delta x$ is the $x$-ordinate distance between the nodes on the $1 \mathrm{D}$ element.

Momentum equation convection terms

The terms $\int_{\Gamma}(h u u) \phi_{i} g_{x} d \Gamma$ and $\int_{\Gamma}(h u v) \phi_{i} g_{y} d \Gamma$ are the $x$-direction momentum boundary line integral terms from Equations 114-115. Equations 143-145 are utilized to integrate these line integrals as follows: 


$$
\begin{gathered}
\int_{\Gamma}(h u u)_{1 e} \phi_{1 e, i} g_{x} d \Gamma=n_{x} \int_{0}^{1} \phi_{1 e, i}(h u u)_{1 e} L d \xi \\
=\Delta y \int_{i 0}^{1} \phi_{1 e, i}(h u u)_{1 e} L d \xi
\end{gathered}
$$

and

$$
\begin{gathered}
\int_{\Gamma}(h u v)_{1 e} \phi_{1 e, i} g_{y} d \Gamma=n_{y} \int_{0}^{1} \phi_{1 e, i}(h u v)_{1 e} L d \xi \\
=-\Delta x \int_{0}^{1} \phi_{1 e, i}(h u v)_{1 e} L d \xi
\end{gathered}
$$

The terms $\int_{\Gamma}(h u v) \phi_{i} \mathrm{~g}_{x} d \Gamma$ and $\int_{\Gamma}(h v v) \phi_{i} \mathrm{~g}_{y} d \Gamma$ are the $y$-direction momentum boundary line integral terms from Equations 116-117. Equations 146-148 are again utilized to integrate these line integrals as follows:

$$
\begin{gathered}
\int_{\Gamma}(h u v)_{1 e} \phi_{1 e, i} g g_{x} d \Gamma=n_{x} \int_{0}^{1} \phi_{1 e, i}(h u v)_{1 e} L d \xi \\
=\Delta y \int_{0}^{1} \phi_{1 e, i}(h u v)_{1 e} L d \xi
\end{gathered}
$$

and

$$
\begin{gathered}
\int_{\Gamma}(h v v)_{1 e} \phi_{1 e, i} g_{y} d \Gamma=n_{y} \int_{0}^{1} \phi_{1 e, i}(h v v)_{1 e} L d \xi \\
=-\Delta x \int_{0}^{1} \phi_{1 e, i}(h v v)_{1 e} L d \xi
\end{gathered}
$$

Momentum equation pressure terms

The term $\int_{\Gamma} \frac{1}{2} \rho g h^{2} \phi_{i} d \Gamma$ are the pressure boundary line integrals in the $x$ and $y$-direction momentum equations. Equations 143-145 are utilized to integrate these line integrals as follows:

For the $x$-momentum equation: 


$$
\begin{gathered}
\int_{\Gamma} \frac{1}{2} \rho g h_{1 e}^{2} \phi_{1 e, i} g_{x} d \Gamma=\frac{1}{2} g \rho n_{x} \int_{0}^{1} \phi_{1 e, i} h_{1 e}^{2} L d \xi \\
=\frac{1}{2} g \rho \Delta y \int_{0}^{1} \phi_{1 e, i} h_{1 e}^{2} L d \xi
\end{gathered}
$$

and for the $y$-momentum equation:

$$
\begin{gathered}
\int_{\Gamma} \frac{1}{2}\left(\rho g h^{2}\right)_{1 e} \phi_{1 e, i} g h_{y} d \Gamma=\frac{1}{2} g \rho n_{y} \int_{0}^{1} \phi_{1 e, i} h_{1 e}^{2} L d \xi \\
=-\frac{1}{2} g \rho \Delta x \int_{0}^{1} \phi_{1 e, i} h_{1 e}^{2} L d \xi
\end{gathered}
$$

All contributions to the FE residuals for all equations have now been detailed. The following section describes boundary conditions in AdH-2D.

\section{Boundary conditions}

Discharge/total flow boundary

AdH-2D allows the user to specify inflow and outflow boundaries at any number of locations on the boundary of the domain. The mathematical and FE treatment of the discharge boundary is similar to the boundary line integrals presented in the previous section and is as follows:

Continuity contribution:

$$
\int_{\Gamma} \phi_{1 e, i} q d \Gamma=L \int_{0}^{1} \phi_{1 e, i} q d \xi
$$

where

$q=$ conveyance capacity dependent upon the flow depth and friction.

$x$-momentum contribution:

$$
\int_{\Gamma} \phi_{1 e, i} u_{1 e} q d \Gamma=L \int_{0}^{1} \phi_{1 e, i} u_{1 e} q d \xi
$$


$y$-momentum contribution:

$$
\int_{\Gamma} \phi_{1 e, i} v_{1 e} q d \Gamma=L \int_{0}^{1} \phi_{1 e, i} v_{1 e} q d \xi
$$

\section{Tailwater or water surface elevation boundary}

AdH-2D allows the user to specify tailwater boundaries at any number of locations on the boundary of the domain. The mathematical and FE treatment of the tailwater boundary is similar to the boundary line integrals presented in the previous section.

\section{Flow per unit length boundary}

AdH-2D allows the user to specify flow per unit width at any number of locations on the boundary of the domain. The mathematical and FE treatment of the discharge boundary is similar to the boundary line integrals presented in the previous section.

\section{Supercritical flow boundary}

AdH-2D allows the user to specify supercritical inflow and/or outflow at any number of locations on the boundary of the domain. The mathematical and FE treatment of the discharge boundary is similar to the boundary line integrals presented in the previous section.

\section{Surface stress terms}

AdH-2d allows the specification of both wind and wave induced stresses into the $x$ - and $y$-direction momentum terms.

\section{Wind-induced surface stress}

AdH-2d provides two options for the calculation of wind stresses: (1) the $\mathrm{Wu}$ (1982) method and (2) the Teeter (2002) method. The stresses are elementally averaged and integrated as follows:

$$
\int_{\Omega} \phi_{i} \tau_{x_{-} \text {wind }} d \Omega=\frac{1}{3} \sum_{i=1}^{3} \tau_{x_{-} \text {wind }, i} 2 \Lambda
$$

and 


$$
\int_{\Omega} \phi_{i} \tau_{y_{-} \text {wind }} d \Omega=\frac{1}{3} \sum_{i=1}^{3} \tau_{y_{-} \text {wind }, i} 2 \Lambda
$$

where

$\tau_{x_{-} \text {wind }}$ and $\tau_{y_{-} \text {wind }}$ are the wind stress components in the $x$ - and $y$-directions respectively.

Wave-induced surface stress

AdH-2d provides two options for the specification of wave stresses: (1) components of the wave stress tensor and (2) wave forces (radiation stresses).

If the components of the wave stress tensor are specified, $\mathrm{AdH}-2 \mathrm{~d}$ computes an elementally averaged applied wave force as follows:

$$
\tau_{x_{-} \text {wave }}-=\sum_{i=1}^{3}\left(\frac{\partial \phi_{i}}{\partial x} \tau_{x x_{-} \text {wave }}+\frac{\partial \phi_{i}}{\partial y} \tau_{x y_{-} \text {wave }}\right)
$$

and

$$
\tau_{y_{-} \text {wave }}-=\sum_{i=1}^{3}\left(\frac{\partial \phi_{i}}{\partial x} \tau_{y x_{-} \text {wave }}+\frac{\partial \phi_{i}}{\partial y} \tau_{y y_{-} \text {wave }}\right)
$$

where $\tau_{x x_{-} \text {wave }}, \tau_{x y_{-} \text {wave }}, \tau_{y y_{-} \text {wave }}$, and $\tau_{y x_{-} \text {wave }}$ are the wave stress components in the $x x-, x y-, y y$-, and $y x$-directions, respectively. The computed wave stress force is then integrated as follows:

$$
\int_{\Omega} \phi_{i} \tau_{x_{-} \text {wave }} d \Omega=\frac{1}{3} \tau_{x_{-} \text {wave }}(2 \Lambda)
$$

and

$$
\int_{\Omega} \phi_{i} \tau_{y_{-} \text {wave }} d \Omega=\frac{1}{3} \tau_{y_{-} \text {wave }}(2 \Lambda)
$$




\section{Determination of model domain extents}

The second terms on the RHS of equation 125 and 126 are the line integrals over the element boundary multiplied by the shape function. These terms are used in AdH-2D to determine the model domain. They are integrated based on $1 \mathrm{D}$ basis functions defined in equation 145 . The integration of these terms involves marching around each line of the computational parent element and adding the contributions to each vertex from the line that includes that vertex, as shown in Figure 11.

Figure 11. 1D line integral basis functions on 2D elements.

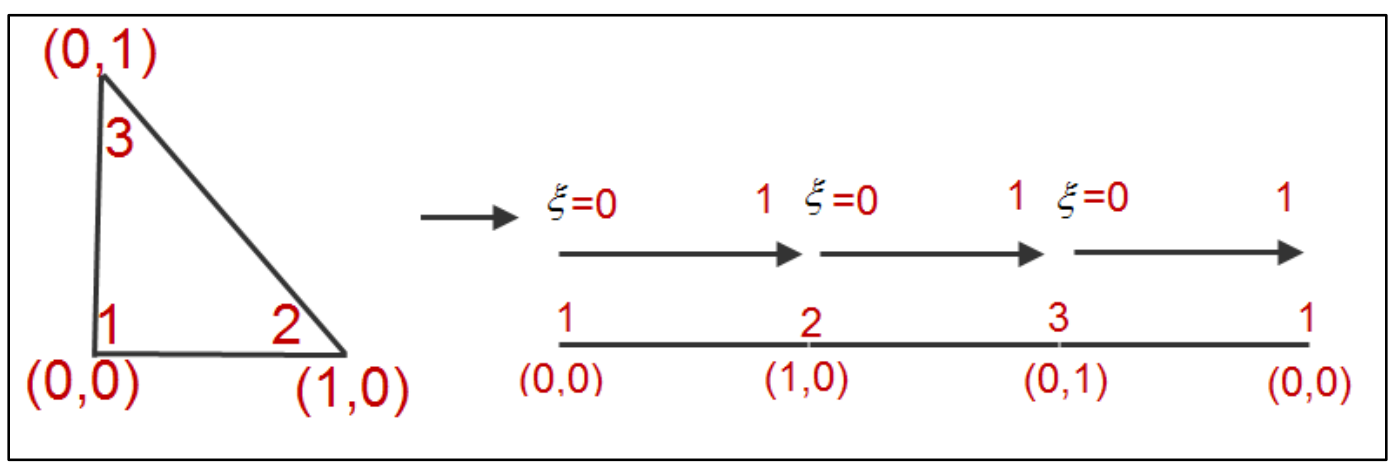

The shape functions, defined in Equation 142, and variable expansions, defined in Equation 143 and 145, are used to integrate the boundary pressure terms to determine the domain no flux boundary.

For the $x$-momentum equation:

$$
\begin{gathered}
\int_{\Gamma} \frac{1}{2} \frac{\rho}{\rho_{0}} g h^{2} \phi_{i} \mathrm{~g}_{x} d \Gamma=\frac{1}{2} g \frac{\rho}{\rho_{0}} n_{x} \int_{0}^{1} \phi_{i} h^{2} L d \xi \\
=\frac{1}{2} g \frac{\rho}{\rho_{0}} \Delta y \int_{0}^{1} \phi_{i} h^{2} L d \xi
\end{gathered}
$$

and for the $y$-momentum equation:

$$
\begin{gathered}
\int_{\Gamma} \frac{1}{2} \frac{\rho}{\rho_{0}} g h^{2} \phi_{i} g_{y} d \Gamma=\frac{1}{2} g \frac{\rho}{\rho_{0}} n_{y} \int_{0}^{1} \phi_{i} h^{2} L d \xi \\
=-\frac{1}{2} g \frac{\rho}{\rho_{0}} \Delta x \int_{0}^{1} \phi_{i} h^{2} L d \xi
\end{gathered}
$$


Where all variables are as previously defined. Note that contribution for a node is added from each line segment that the node is a part of.

This integration is done on a $2 \mathrm{D}$ element using the $1 \mathrm{D}$ element that comprises the element. On an interior edge, the $1 \mathrm{D}$ element is adjacent to two active elements, each producing an equal and opposite contribution, resulting in no RHS contribution for this edge. On a boundary edge, however, there will be a contribution from the pressure boundary. In this way, the model implicitly finds no-flux boundaries without any explicit domain boundary specification from the user.

\section{Petrov-Galerkin (PG) terms}

Equation 28 mathematically represents the PG terms used in the discontinuous part of the AdH-2D equation set. This equation, presented again, is the following:

$$
\overline{\mathbf{P}}_{i} \equiv\left\{\begin{array}{l}
\bar{P}_{c}^{i} \\
\bar{P}_{m x}^{i} \\
\bar{P}_{m y}^{i}
\end{array}\right\}=\int_{\Omega_{e}} \tau^{e}\left[\mathbf{A}_{x}^{e} \frac{\partial \phi_{i}}{\partial x}+\mathbf{A}_{y}^{e} \frac{\partial \phi_{i}}{\partial y}\right] \mathbf{H}^{e} \overline{\mathbf{R}}_{n c}^{*} d \Omega_{e}
$$

where $\tau^{e}\left[\mathbf{A}_{x}^{e} \frac{\partial \phi_{i}}{\partial x}+\mathbf{A}_{y}^{e} \frac{\partial \phi_{i}}{\partial y}\right]=\varphi$ is the discontinuous part of the AdH-2D test function and all terms are as previously defined.

The PG terms, in general, are integrated as elementally averaged values times the discontinuous part of the AdH-2D test function, $\varphi$.

Note that as previously stated, the discontinuous part of the test function is only integrated within interior of the element and therefore includes no boundary terms. Boundary terms, as previously described, are taken care of through the continuous part, $\phi$, of the test function.

If the following are defined, where the superscript " $e$ " denotes sum over the elemental and where the subscripts " $x, y$ " denote gradient in the $x$ and $y$, respectively, 


$$
\begin{aligned}
h_{x}^{e} & =\sum_{i=0}^{2} h_{i} \frac{\partial \phi_{i}}{\partial x} \\
u_{x}^{e} & =\sum_{i=0}^{2} u_{i} \frac{\partial \phi_{i}}{\partial x} \\
v_{x}^{e} & =\sum_{i=0}^{2} v_{i} \frac{\partial \phi_{i}}{\partial x} \\
z_{x}^{e} & =\sum_{i=0}^{2} z_{i} \frac{\partial \phi_{i}}{\partial y} \\
h_{y}^{e} & =\sum_{i=0}^{2} h_{i} \frac{\partial \phi_{i}}{\partial y} \\
u_{y}^{e} & =\sum_{i=0}^{2} u_{i} \frac{\partial \phi_{i}}{\partial y} \\
v_{y}^{e} & =\sum_{i=0}^{2} v_{i} \frac{\partial \phi_{i}}{\partial y} \\
z_{y}^{e} & =\sum_{i=0}^{2} z_{i} \frac{\partial \phi_{i}}{\partial y}
\end{aligned}
$$

The PG terms can be written as follows:

\section{Continuity equation}

$$
\bar{P}_{c}^{i}=\int_{\Omega_{e}} \tau^{e}\left[\mathbf{A}_{x}^{e} \frac{\partial \phi_{i}}{\partial x}+\mathbf{A}_{y}^{e} \frac{\partial \phi_{i}}{\partial y}\right]\left(\frac{\partial h^{e}}{\partial t}+\bar{u} h_{x}^{e}+\bar{v} h_{y}^{e}\right) d \Omega_{e}
$$

x-direction momentum equation

$$
\bar{P}_{m x}^{i}=\int_{\Omega_{e}} \tau^{e}\left[\mathbf{A}_{x}^{e} \frac{\partial \phi_{i}}{\partial x}+\mathbf{A}_{y}^{e} \frac{\partial \phi_{i}}{\partial y}\right]\left(\begin{array}{l}
\frac{\partial(u h)^{e}}{\partial t}+\bar{h} \bar{u} u_{x}^{e}+\bar{h} v u_{y}^{e}-f \bar{h} \bar{v}+ \\
\frac{\rho}{2 \rho_{0}} g \bar{h}+\frac{\rho}{2 \rho_{0}} g z_{x}^{e}+\frac{\overline{\tau_{x_{-} s}}}{\rho_{0}}
\end{array}\right) d \Omega_{e}
$$

y-direction momentum equation

$$
\bar{P}_{m y}^{i}=\int_{\Omega_{e}} \tau^{e}\left[\mathbf{A}_{x}^{e} \frac{\partial \phi_{i}}{\partial x}+\mathbf{A}_{y}^{e} \frac{\partial \phi_{i}}{\partial y}\right]\left(\begin{array}{l}
\frac{\partial(v h)^{e}}{\partial t}+\bar{h} \bar{u} v_{x}^{e}+\bar{h} v v_{y}^{e}+f \bar{h} \bar{u}+ \\
\frac{\rho}{2 \rho_{0}} g \bar{h} \bar{h}+\frac{\rho}{2 \rho_{0}} g \bar{h} z_{y}^{e}+\frac{\overline{\tau_{y_{-} s}}}{\rho_{0}}
\end{array}\right) d \Omega_{e}
$$

Where all variables are as previously defined and the overbar indicates an elemental average. 


\section{Appendix C: Friction Library}

AdH-2D has the capability to represent the coefficient of friction, $C_{f}$, using a number of physically relevant formulations to accurately account for friction losses.

\section{Bed shear coefficient of friction}

The bed shear formulation computes a shear stress coefficient resulting from a steady (or quasi-steady) current field. The formulation given here is derived from a modified form of the classic logarithmic velocity profile. This modified profile was physically justified by Christensen (1972). The traditional profile yields a velocity of $-\infty$ at the bed whereas the modified profile forces the velocity to $\mathrm{o}$ at the bed. The formulation is as follows:

$$
\begin{gathered}
C_{f}=2\left(\frac{\kappa \beta}{[(\beta+1)\{\ln (\beta+1)-1\}+1]}\right)^{2} \\
\beta=29.7 \frac{h}{k} \\
\kappa=0.4
\end{gathered}
$$

where $C_{f}$ is the bed shear stress drag coefficient, $h$ is the water depth, $k$ is the equivalent sand roughness height, and $\kappa$ is the Von Kárman constant.

\section{Submerged aquatic vegetation (SAV) coefficient of friction}

The SAV formulation will compute a shear stress coefficient for use in computing the bottom shear stress resulting from a steady (or quasisteady) current field over a bed consisting of SAV. The formulation given here is from Christensen (1985) with average vegetation characteristics taken from Jacobs and Wang (2003).

The shear stress is computed as follows:

$$
C_{f}=2\left(\frac{\kappa \beta}{[(\beta+\lambda)\{\ln (\beta+\lambda)-1\}+1]}\right)^{2}
$$




$$
\begin{gathered}
\beta=29.7 \frac{h}{k} \\
\lambda=1-29.7 \frac{t}{k} \\
t \cong \frac{2}{3} h_{\text {sav }} \\
\kappa=0.4
\end{gathered}
$$

where all variables are as previously defined, $t$ is the apparent thickness of the near-zero velocity region induced by the presence of the $\mathrm{SAV}, h_{\text {sav }}$ is the undeflected stem height of the SAV, and $\kappa$ is the Von Kárman constant.

The approximate value given for $t$ as a function of $h_{s a v}$ is taken from Jacobs and Wang (2003). A recommended value of $k$, also taken from this source, is given as follows:

$$
k \cong \frac{1}{10} h_{\text {sav }}
$$

\section{Unsubmerged rigid vegetation coefficient of friction}

The unsubmerged rigid vegetation formulation will compute a shear stress coefficient for use in computing the bottom shear stress resulting from a steady (or quasi-steady) current through rigid, unsubmerged vegetation. Some examples of this might include flow through mangrove stands, through phragmites in coastal wetlands, or through trees and other obstructions in coastal storm surge flooding. The formulation given here is taken from Walton and Christensen (1980). This formulation includes the form drag induced by flow through the obstructions and the skin drag induced by flow over the bed.

$$
\begin{gathered}
C_{f}=\frac{0.32\left(1-m \frac{\pi}{4} \delta^{2}\right)}{\left[\ln \left(\frac{10.94 h}{k}+1\right)\right]^{2}}+C_{f . S}(h)(m)(\delta) \\
C_{f . S}=0.4
\end{gathered}
$$

where $C_{f . S}$ is the drag coefficient for the stems, $\delta$ is the average stem diameter, and $m$ is the average stem density. 


\section{Evenly distributed obstruction coefficient of friction}

The evenly distributed obstruction formulation will compute a shear stress coefficient for use in computing the shear stress resulting from a steady (or quasi-steady) current through or over an evenly distributed field of flow obstructions. This formulation can be used to simulate flow through or over wetland vegetation, trees, buildings, or any other subgrid-scale obstructions. The obstructions are modeled as a field of evenly distributed cylinders.

The formulation given here is a combination of the UNsubmegedRigidVegatation formulation taken from Walton and Christensen (1980) and the SubmergedAquaticVegetation formulation taken from Christensen (1995) and Jacobs and Wang (2003).

To apply this formulation, first, a drag coefficient is computed with the assumption that the obstructions are unsubmerged. Then, a drag coefficient is computed with the assumption that the obstructions are submerged. The final computed drag coefficient is selected to be the minimum of these two values.

The drag coefficient associated with unsubmerged obstructions is given as

$$
\begin{gathered}
C_{f . U}=\frac{0.32\left(1-m \frac{\pi}{4} \delta^{2}\right)}{\left[\ln \left(\frac{10.94 h}{k_{B}}+1\right)\right]^{2}}+C_{f . S O}(h)(m)(\delta) \\
C_{f . S O}=0.4
\end{gathered}
$$

where $C_{f . U}$ is the bed shear stress coefficient for unsubmerged conditions, $h$ is the water depth, $k_{B}$ is the equivalent sand roughness height of the bed, $C_{f . S O}$ is the coefficient for a single obstruction, $\delta$ is the average obstruction diameter, and $m$ is the average obstruction density.

The coefficient of friction associated with submerged obstructions is given as follows: 


$$
\begin{gathered}
C_{f . S}=2\left(\frac{\kappa \beta}{[(\beta+\lambda)\{\ln (\beta+\lambda)-1\}+1]}\right)^{2} \\
\beta=29.7 \frac{h}{k_{C}} \\
\lambda=1-29.7 \frac{t}{k_{C}} \\
t \cong \frac{2}{3} h_{O B S} \\
\kappa=0.4
\end{gathered}
$$

where $C_{f . S}$ is the bed shear stress drag coefficient associated with submerged obstructions, $h$ is the water depth, $k_{C}$ is the equivalent sand roughness height of the obstruction canopy (when they are submerged), $t$ is the apparent thickness of the near-zero velocity region near the bed induced by the presence of the obstructions, hoBs is the average height of the obstructions, and $\kappa$ is the Von Kárman constant. The approximate value given for $t$ as a function of hoвs is taken from Jacobs and Wang (2003). 


\section{Acronyms and Abbreviations}

2D two-dimensional

AdH Adaptive Hydraulics

FE finite element

PG Petrov-Galerkin

PTC Pseudo-Transient Computation

RANS Reynolds Averaged Navier-Stokes

RHS right-hand side

SAV submerged aquatic vegetation

SER Switched Evolution Relaxation

SUPG Streamline Upwind Petrov-Galerkin

SW shallow water

USACE US Army Corps of Engineers 


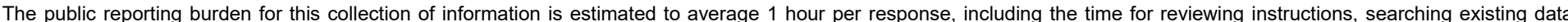

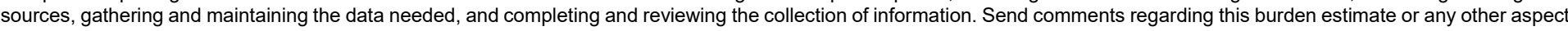

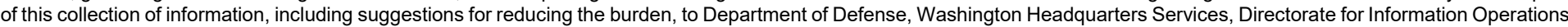

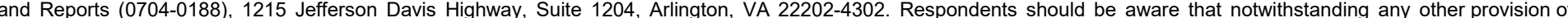
law, no person shall be subject to any penalty for failing to comply with a collection of information if it does not display a currently valid OMB control number. PLEASE DO NOT RETURN YOUR FORM TO THE ABOVE ADDRESS.

\begin{tabular}{l|l|l}
$\begin{array}{l}\text { 1. REPORT DATE } \\
\text { May } 2020\end{array}$ & $\begin{array}{l}\text { 2. REPORT TYPE } \\
\text { Final Report }\end{array}$ & 3. DATES COVERED (FrOm - To)
\end{tabular}

\section{TITLE AND SUBTITLE}

Theory, Formulation, and Implementation of The Cartesian and Spherical Coordinate Two-

Dimensional Depth-Averaged Module of the Adaptive Hydraulics (AdH) Finite Element

Numerical Code

5a. CONTRACT NUMBER

5b. GRANT NUMBER

5c. PROGRAM ELEMENT NUMBER

\section{AUTHOR(S)}

5d. PROJECT NUMBER

Gaurav Savant, Charlie Berger, Corey J. Trahan, and Gary L. Brown

5e. TASK NUMBER

5f. WORK UNIT NUMBER

\section{PERFORMING ORGANIZATION NAME(S) AND} ADDRESS(ES)

Coastal and Hydraulics Laboratory

US Army Engineer Research and

Development Center

3909 Halls Ferry Road

Vicksburg, MS 39180-6199

\section{SPONSORING/MONITORING AGENCY NAME(S) AND ADDRESS(ES)}

Flood and Coastal Systems Research and Development Program

US Army Engineer Research and Development Center

3909 Halls Ferry Road

Vicksburg, MS 39180-6199
Information Technology Laboratory

US Army Engineer Research and

Development Center

3909 Halls Ferry Road

Vicksburg, MS 39180-6199

\section{DISTRIBUTION/AVAILABILITY STATEMENT}

Approved for public release; distribution is unlimited.

\section{SUPPLEMENTARY NOTES}

Funding Acct Code U4354615; Work Unit Adaptive Hydraulics (AdH) Development, Verification, and Validation

\section{ABSTRACT}

The US Army Engineer Research and Development Center, Coastal and Hydraulics Laboratory, has undertaken the development of the multi-module Adaptive Hydraulics (AdH) hydrodynamic, sediment, water quality, and transport numerical code. This report documents the mathematical formulation and numerical implementation of the two-dimensional depth-averaged module of AdH.

\section{SUBJECT TERMS}

Finite element method, Hydraulics-Mathematical models, Hydrodynamics_-Mathematical models, Numerical analysis, Sediment transport-Mathematical models

16. SECURITY CLASSIFICATION OF:

\begin{tabular}{|l|c|l|}
\hline a. REPORT & b. ABSTRACT & c. THIS PAGE \\
Unclassified & Unclassified & Unclassified \\
\hline
\end{tabular}
ABSTRACT

SAR
18. NUMBER 19a. NAME OF RESPONSIBLE PERSON OF PAGES

73 Gaurav Savant

19b. TELEPHONE NUMBER (Include area code) 601-634-3213
8. PERFORMING ORGANIZATION REPORT NUMBER ERDC TR-20-8

10. SPONSOR/MONITOR'S ACRONYM(S) F\&CS R\&D ERDC

1. SPONSOR/MONITOR'S REPORT NUMBER(S) 